\title{
Landschaftsorganisation, Siedlungsnetz und Dorfstruktur in der Germania in den Jahrzehnten um Christi Geburt
}

\section{Vorbemerkungen}

Während der Tagung „Römische Präsenz und Herrschaft in Germanien der augusteischen Zeit“ im Jahr 2004 habe ich über ein ähnliches Thema gesprochen und kann einige Ergebnisse hier deshalb knapp zusammenfassen. ${ }^{1}$

Ich habe erläutert, dass die Besiedlungsdichte in den ersten Jahrhunderten n. Chr. in der Germania beachtlich groß war und dass die Dörfer aus 10 und mehr Gehöften oft in Blickverbindung zueinander lagen. Ein ausgebautes Wegenetz verband die Siedlungen miteinander. Die aufgrund der archäologischen Befunde errechneten Bevölkerungsgrößen und die daraus erschließbaren Heeresstärken zeigen, dass den römischen Legionen ohne Schwierigkeiten beachtliche germanische Militärverbände entgegengestellt werden konnten.

Meine These war und ist, dass deshalb schließlich auch der Versuch Roms aufgegeben wurde, die Germania zur Provinz umzugestalten. Mächtiger Gegendruck und die andersartige zivilisatorische Struktur schlossen eine vergleichbare Eroberung wie in der Gallia mit ihren Zentralorten aus, die besetzt werden konnten und womit die Oligarchien der Stämme in den Griff zu bekommen waren. Man kann sich die Lage im damaligen Germanien gut vorstellen, wenn man als Analogie die gegenwärtigen Verhältnisse in umkämpften, zerfallenden Staaten beobachtet: Sie bilden nicht nur geschlossene Territorien mit Grenzen und einer entsprechend verteilt siedelnden Bevölkerung, sondern die Länder werden überlagert von beweglichen kriegerischen Personengruppen aus ehemaligen Stammesverbänden unter Kriegsherren/Warlords, die wechselnde Areale besetzen und mit denen kaum dauerhaft Verträge zu schließen sind.

Das Thema bleibt aber zu diskutieren, und ich habe im Titel die Hierarchie formuliert: Dorfstruktur, Siedlungsgefüge und gesamte Landschaftsorganisation. ${ }^{2}$ Auch frühgeschichtliche Landschaften sind nicht einfach Ansammlungen von Siedlungsnetzen samt Wegeverbindungen zwischen den Dörfern, sondern nicht nur nach Meinung der Forschung soziale und mentale Konstrukte. ${ }^{3}$

1 Steuer 2007.

2 Siedlungs-, Gehöft- und Hausformen 2005. - Ähnlich jetzt die Habilitationssschrift von Hans-Jürgen Nüsse 2014, oder auch Meyer (Hrsg.) 2010: Haus - Gehöft - Weiler - Dorf .

3 Settlement and Landscape 1999, S. 329 als Überschrift für eine Sammlung von Beiträgen: „The landscape seen as a social and mental construct“, S. 331 „Ordering landscapes“ (Herschend 1999), 
Wie waren die Siedlungen, das Siedlungsnetz und die Landschaft zur Zeit der römischen Invasionen strukturiert, wie haben sich Herrschaft und Stammesgruppierungen in der Landschaft manifestiert? Worauf stießen die römischen Truppen beim Marsch durch die Landschaften? Es zeigt sich, dass die militärische Dominanz Roms sich nicht so unmittelbar im archäologisch fassbaren Siedlungsbild ausdrückt. Manche Kartenbilder suggerieren aber, dass Rom auf dem Weg war, Norddeutschland zur Provinz zu machen; Einfärbungen beziehen entweder die Landschaften südlich der Nordseeküste mit ein oder sogar alle Gebiete bis zur Weser. ${ }^{4}$

Aufgrund des unterschiedlichen archäologischen Forschungsstandes kann man auch nicht nur die beginnenden Mittelgebirgszone, also von Kalkriese bis Hedemünden betrachten, sondern es muss erlaubt sein, für analogisches Vergleichen weiter nach Norddeutschland und Jütland sowie in die Niederlande auszugreifen, weil dort die Zahl und der Umfang der ausgegrabenen Siedlungen wesentlich größer ist als im Binnenland.

Den Forschungsstand in Südniedersachsen fasst Klaus Grote in seinem Buch über das Römerlager Hedemünden zusammen, ergänzt durch eine Karte (Abb. 1 entspricht Abb. 11 des Aufsatzes von Klaus Grote im Farbteil). ${ }^{5}$ Der Süd-Nord verlaufende, $40 \mathrm{~km}$ lange und bis $10 \mathrm{~km}$ breite Leinetalgraben war eine offene, flächig besiedelte Landschaft. Die nachgewiesenen Siedlungsplätze liegen dicht an dicht, nur wenige Kilometer auseinander. Aber: „Allerdings liegen aus fast allen genannten Orten keine oder nur sehr ausschnitthafte, zudem ältere Untersuchungen vor, sodass hier vermutlich auf lange Zeit keine detaillierten Aussagen möglich sind““. ${ }^{6}$ Die Landschaft machte es den römischen Truppen beim Durchmarsch durch das weitgehend gerodete und verkehrsoffene Gebiet leicht, ${ }^{7}$ weil alle Möglichkeiten zum Fouragieren, Plündern und Zerstören der maßgeblichen Siedlungen wie Dörfer, Burganlagen (?) und andere Zentral-/Kultorte gegeben waren. ${ }^{8}$

Die Situation bei Kalkriese ist ähnlich. Zu Füßen des Berges verlief seit eh und je und auch weiterhin ein viel begangener Verkehrsweg. ${ }^{9}$ Und nahebei gab es in der fruchtbaren Landschaft germanische Siedlungen, die hier wenigstens teilweise archäologisch erschlossen sind, und Joachim Harnecker meint: „Kalkriese war kein germanischer Urwald - Was Siedlungsfunde über den Ort des Schlachtgeschehens verraten“ ${ }^{10}$ Mehrere Siedlungsplätze, die mit Sicherheit zur Zeit der Schlacht bestan-

4 Beispielsweise Eck 2009, S. 143 Abb. 2.

5 Grote u.a. 2012, S. 310 ff. mit Karte Abb. 325.

6 Grote u.a. 2012, S. 311 Anm. 42.

7 Das Klischee vom undurchdringlichen germanischen Urwald zieht sich bis in die neueste wissenschaftliche Literatur, vgl. dazu Steuer 2007, S. 337 und neu Fried 2014, S.10. - Was man unter umfassender Bewaldung versteht, ist relativ: Heute ist Deutschland immerhin zu einem Drittel noch und wieder mit Wald bedeckt

8 Grote u.a. 2012, S. 311.

9 Schlüter / Wiegels 2000.

10 Harnecker 2009, S. 89-91 mit Karte S. 88. 
den, sind archäologisch in der Hangsandzone am Fuß des Kalkrieser Berges erfasst (Abb. 2).

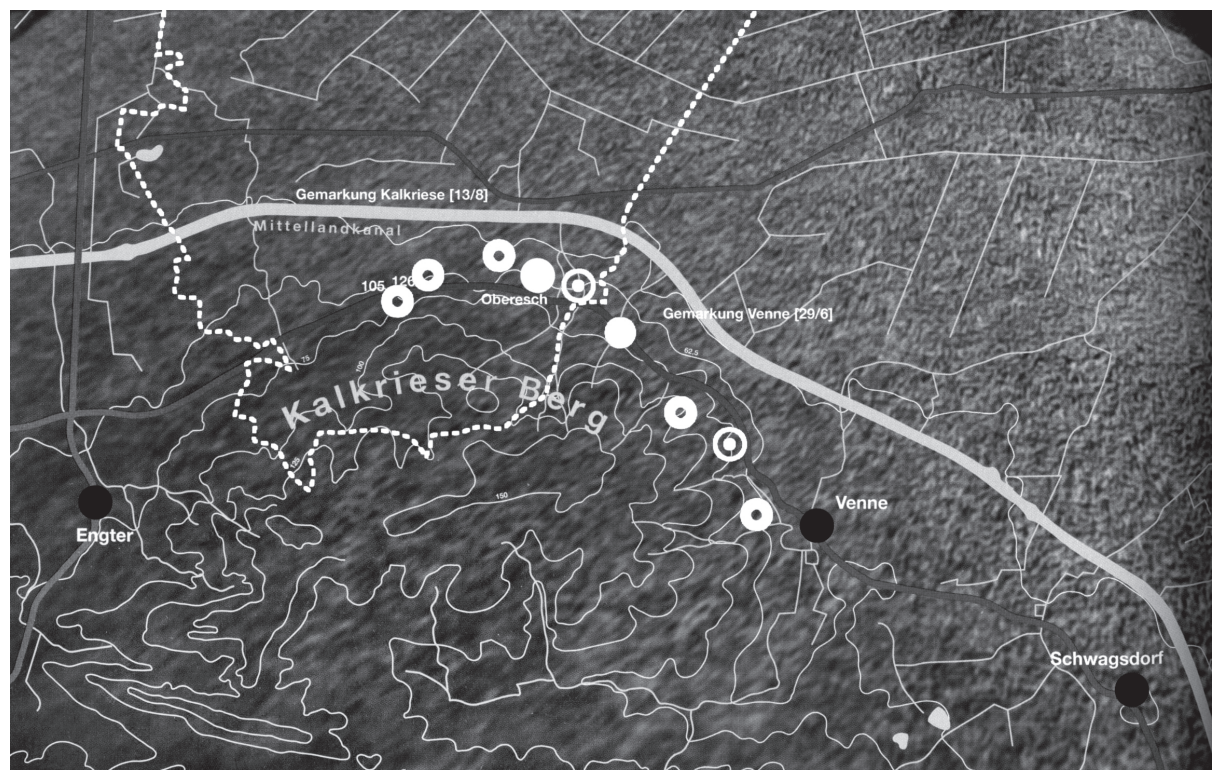

Abb. 2: Siedlungen zu Füssen des Kalkrieser Berges während der späten Eisenzeit und der frühen Römischen Kaiserzeit (nach Harnecker 2009, S.88 Abb. 1 - verändert).

In der Siedlung beim Hof Dröge wurden zudem Fundmaterialien und Metallschrott geborgen, die zeigen, dass hier Plünderungsgut von Schlachtfeldern weiterverarbeitet wurde. ${ }^{11}$ Einige zweischiffige Hausgrundrisse der Siedlung Dröge sind dokumentiert. Nicht weit entfernt ist zuvor schon bei Engter, Stadt Bramsche, eine germanische Siedlung ausschnitthaft ausgegraben worden, ${ }^{12}$ wobei jedoch immerhin Hausgrundrisse von 15 bis 20 m Länge erkannt werden konnten, ebenfalls zumeist zweischiffig. Jürgen Pape hat seinerzeit eine Karte erstellt, die zwei Hauslandschaften in der Germania abbildet: ${ }^{13}$ Im Süden zum Mittelgebirge hin dominieren zweischiffige Häuser - also mit einer Pfostenreihe in der Mitte unter dem Giebelverlauf -, deren Verbreitung sich auch weit nach Westen über den Rhein ins Gebiet der Bataver erstreckt. Im Norden zur Küste hin herrschen die dreischiffigen Wohn-Stall-Häuser vor.

11 Harnecker 2009, S. 91 mit Abb. 3. - Wie die Kommunikation im Netz der germanischen Siedlungen funktioniert hat, belegen die Fragmente der Reiterstatue aus der römischen „Stadtgründung“ Waldgirmes, die in verschiedenen Dörfern der näheren und weiterern Umgebung ausgegraben worden sind: Schäfer / Schroth 2009; Schnurbein 2011, S. 95-97.

12 Pape 1993, S. 307-321, hier S. 309 mit Abb. 3.

13 Pape 1993, S. 313 mit Abb. 4. 


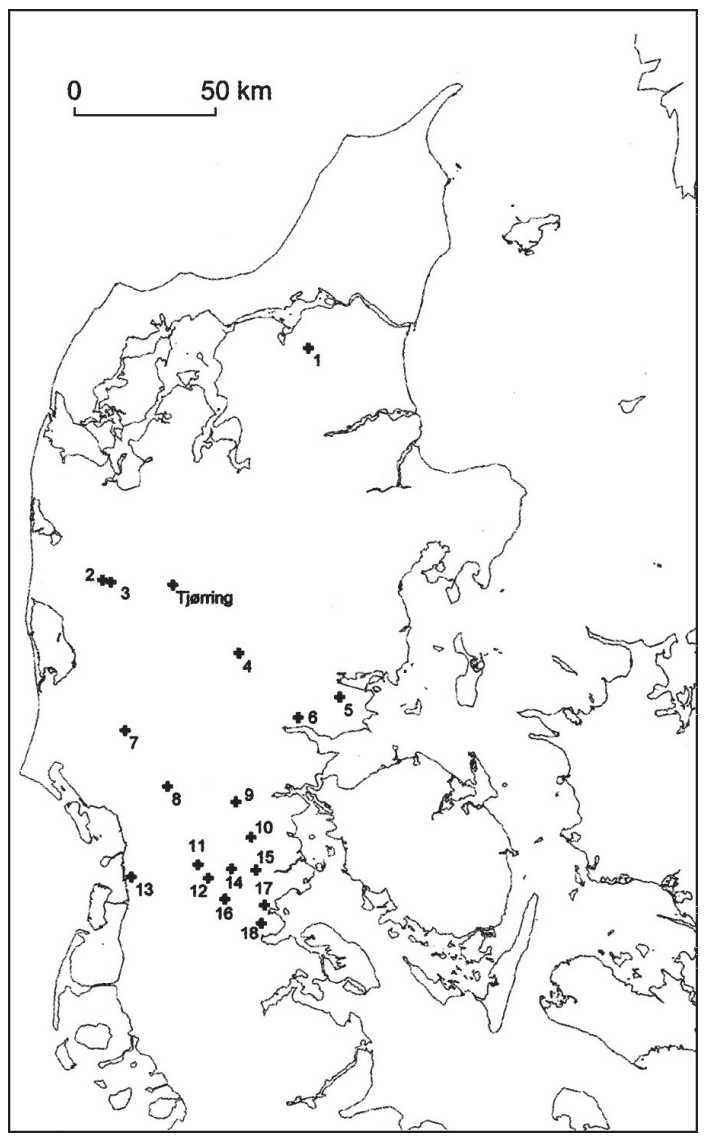

Abb. 3: Archäologisch erforschte Siedlungen der späten Eisenzeit und Römischen Kaiserzeit in Jütland (nach Møller-Jensen 2010, S. 198 Fig. 1 - verändert).

Aber alle diese Befunde reichen nicht aus, um ein einigermaßen vollständiges Bild des Siedlungsmusters in der Germania zu gewinnen. Deshalb ist ein Blick auf die Küstenzone, nach Jütland und in die Niederlande notwendig. Die Zahl meist fast vollständig ausgegrabener Siedlungen, wobei Flächen von 15 bis 20 Hektar abzudecken waren, ist in den wenigen letzten zehn Jahren außerordentlich angestiegen, so dass die älteren zusammenfassenden Publikationen von Georg Kossack ${ }^{14}$ oder der Göttinger Akademie-Kommission ${ }^{15}$, beide aus dem Jahr 1997, schon deutlich überholt sind. Für das mittlere Jütland zeigt eine Karte aus dem Jahr 2010, zusammengestellt von Erik Møller-Jensen, wie die ausgegrabenen Siedlungsplätze geballt in oft weniger als 5-10 km Entfernung auseinander liegen (Abb. 3). ${ }^{16}$ Für dänische Landschaften gibt es

14 Kossack 1997.

15 Beck / Steuer (Hrsg.) 1997, darin: Sten Hvass, The Status of the Iron Age Settlement in Denmark, S. 377-413, und W. Haio Zimmermann, Haus, Hof und Siedlungsstruktur auf der Geest vom Neolithikum bis in das Mittelalter im Elbe-Weser-Dreieck, S. 414- 460.

16 Møller-Jensen 2010, S. 198 Fig. 1. 
weitere Kartierungen, die zeigen, in welch geringem Abstand die Siedlungen der vorrömischen Eisenzeit und der Römischen Kaiserzeit lagen; ${ }^{17}$ und es gibt - nur als ein Beispiel -auch für das Havelland in Deutschland eine solche Kartierung. ${ }^{18}$

Es fällt nun leider auf, dass anders als in der Küstennähe zur Nordsee weiter im Binnenland seltener Siedlungen der Zeit um Chr. Geb. untersucht worden sind. Es liegen meist nur Ausschnitte einer Siedlung vor, kaum jedoch eine Gesamterforschung wie in Jütland oder dem nördlichen Niedersachsen und den Niederlanden.

Die jährlichen Berichte der Archäologischen Bodendenkmalpflege bringen stattdessen - wegen anderer Faszinierung - häufiger Ergebnisse zu römischen Siedlungsgrabungen, Villenuntersuchungen oder Lagererforschungen, so beiderseits des Rheins und im westlichen Westfalen.

\section{Befestigungen und „Herrenhöfe ${ }^{\text {c19 }}$}

Auf dem Rückmarsch von seinem Feldzug gegen die Chatten im Jahr 15 n. Chr. kam Germanicus dem Hilferuf des Segestes nach, der von Arminius belagert wurde.

Wenig später trafen Gesandte von Segestes ein mit der Bitte um Hilfe gegen die Übermacht seiner Landsleute, von denen er eingeschlossen wurde; die stärkere Stellung bei ihnen besaß ja Arminius... Dem Germanicus war es der Mühe wert umzukehren; es kam zu einem Kampf mit den Belagerern und zur Befreiung des Segestes samt einer großen Schar von Verwandten und Gefolgsleuten. Dabei waren vornehme Frauen, darunter die Gattin des Arminius und Tochter des Segestes... Man brachte auch Beutestücke von der Niederlage des Varus, die zumeist den Kriegern, die sich jetzt unterwarfen, als Beuteanteil gegeben worden waren... (Tac. Ann. I 57). ${ }^{20}$

Zwar wird nicht gesagt, worin oder wie sich Segestes verschanzt hatte bzw. wie die Belagerung organisiert war. Ich gehe aber von einem größeren befestigten Gehöft aus. Der knappe Text spricht von einer höheren Zahl von Menschen, genannt werden die Familie, Gefolgsleute des Segestes und Krieger, die belagert und von Germanicus befreit wurden. Wie soll man sich dieses herrschaftliche Anwesen des Segestes vorstellen, das für so viele Menschen Schutz bot, nicht leicht erobert werden konnte, sondern belagert werden musste.

17 Kaldal Mikkelsen 1999, S. 184 Fig. 5: Siedlungsplätze der späten vorrömischen Eisenzeit; weitere Abb. für Jütland bei Steuer 2007, S. 350 mit Abb. 6.1 (nach Rindel 1999, S. 82 Fig).

18 Steuer 2007, S. 351 Abb. 6 (nach May 2002, S. 33 Abb.).

19 Bederkesa 2010.

20 Neque multo post legati a Segeste venerunt auxilium orantes adversus vim popularium, a quis circumsedebatur, validiore apud eos Arminio... Germanico pretium fuit convertere agmen, pugnatumque in obsidentis, et ereptus Segestes magna cum propinquorum et clientium manu. Inerant feminae nobiles, inter quas uxor Arminii eademque filia Segestis... ferebantur et spolia Varianae cladis, plerisque eorum qui tum in deditionem veniebant praedae data (Heller 1982, S. 82 f.). 
Das Gehöft scheint nicht nur aus einem großen, von einem Zaun oder einer Palisade umgebenen, Haus bestanden zu haben, sondern wird stärker befestigt gewesen sein. Wie sehen dazu archäologische Befunde aus?

Mehr oder weniger stark befestigte Großhöfe hat es in vielen Perioden der Ur- und Frühgeschichte gegeben, solche aus der frühen Römischen Kaiserzeit wären somit nichts Besonderes. Aus der Hallstattzeit (bis 450 v. Chr.) sind - ebenfalls erst in den letzten Jahrzehnten - hunderte von rechteckigen Herrenhöfen entdeckt worden, deren Verbreitung kartiert wurde. ${ }^{21}$ Viele sind auch ausgegraben worden. Bis zu drei und fünf Palisaden- und Grabenzüge hegen die Höfe ein, und auf der Fläche standen mehrere Wohn- und Wirtschaftsbauten. Auch für die Latènezeit (bis gegen 50 v. Chr.) sind ähnliche Gehöftplätze in dichter Verbreitung in Süddeutschland und Nordfrankreich nachgewiesen. Sie werden als Viereckschanzen ${ }^{22}$ bezeichnet, wegen des öfter noch erhaltenen Walles, wurden früher als Kultanlagen gedeutet, haben aber jetzt nach zahlreichen Ausgrabungen erkennen lassen, dass es sich um landwirtschaftliche Gehöfte gehobenen Ranges handelt, die entweder abseits oder aber auch inmitten sonstiger Gehöftverteilung liegen. Aus der späten Latènezeit sind weiterhin im Norden, beiderseits des Niederrheins befestigte Siedlungen mit zahlreicher Innenbebauung nachgewiesen.

So gibt es räumliche und zeitliche Zusammenhänge zu den ebenfalls befestigten Herren-Gehöften im germanischen Norden. Hier sind sie aber bisher sehr viel seltener entdeckt oder ausgegraben worden. Großsiedlungen wie die Oppida, oftmals Höhenbefestigungen, werden allgemein als „keltisch“ angesprochen; sie reichen aber fast ins „germanische“ Gebiet hinein, bis zum Nordrand der Mittelgebirge in Mitteleuropa, was aus topographischen Gründen naheliegend ist, so dass schon deshalb mögliche Befestigungen im Flachland aus der späten vorrömischen Eisenzeit anders strukturiert und gebaut sein müssten.

Die Schnippenburg im Landkreis Osnabrück, datiert ins 3.-2. Jahrhundert, ist eine mittellatènezeitliche Befestigung mit umfassend belegten Kultaktivitäten. ${ }^{23}$ Die Pipinsburg bei Osterode im Südharz ist nach frühen Phasen ebenfalls in der Mittellatènezeit als Befestigung mit Oppidum-Charakter zu werten. ${ }^{24}$ Dass sich am Rande der Mittelgebirgszone auch eine kulturelle Grenze festmachen lässt, spiegelt z.B. die Verbreitung bestimmter Grabformen, wie sie Bernhard Sicherl ${ }^{25}$ und Wolfgang Schlüter ${ }^{26}$ kartiert haben, oder wie Frank Verse anhand der Verbreitung keltischer Münzen der Mittel- und Spätlatènezeit zeigen kann, die nicht weiter nach Norden vorkommen. ${ }^{27}$ Die römischen Armeen zogen also teilweise durch Landschaften, deren

21 Berg-Hobohm 2010.

22 Wieland 2006; Büchsenschütz / v. Nicolai 2012, S. 382 mit Verbreitungskarte.

23 Möllers 2007; Schlüter 2004.

24 Schlüter 2003; Caselitz / Schlüter 2013.

25 Sicherl 2007, S. 133 Abb. 19.

26 Schlüter 2007, z.B. S. 295 Abb. 2 und S. 297 Abb. 4.

27 Verse 2007, S. 160 Abb. 10. 
Zugehörigkeit, wenn man mit solchen alten ethnischen Bezeichnungen noch arbeitet, zum keltischen oder zum germanischen Siedlungsraum unklar oder wechselnd ist. Das zeigt auch deutlich eine Karte der Kulturgruppen-Verteilung am Ende der vorrömischen Eisenzeit (Abb. 4, siehe Farbteil). ${ }^{28}$

Eigentlich weiß man sehr wenig von Befestigungen oder Burgen bei den Germanen. ${ }^{29}$ Eine erste Karte bietet Jes Martens (Abb. 5). ${ }^{30}$

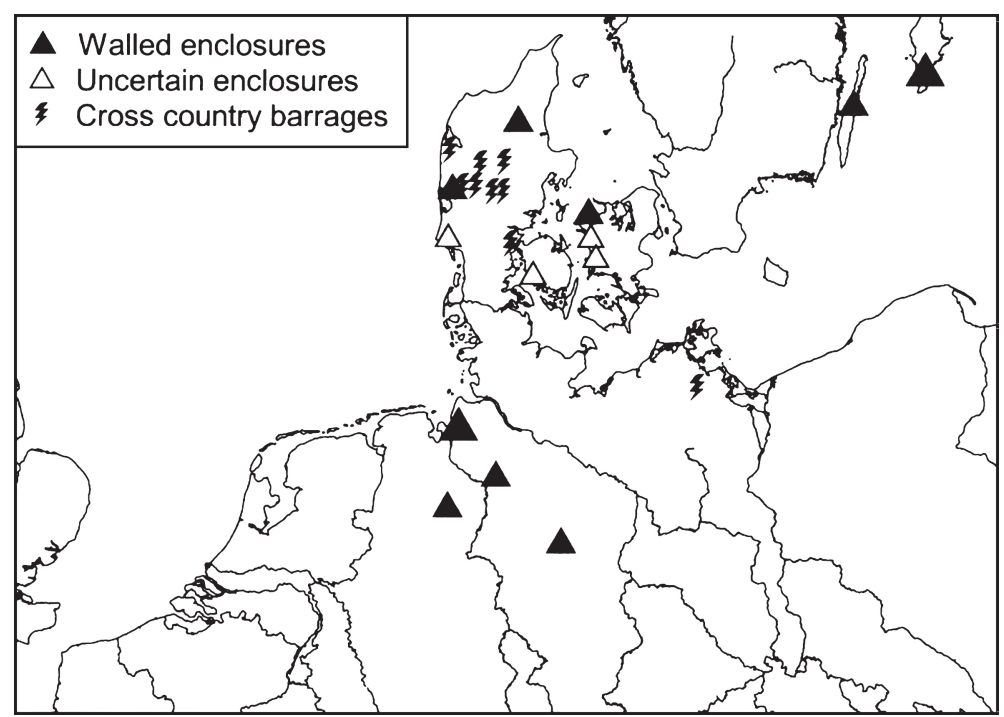

Abb. 5: Wallanlagen der vorrömischen Eisenzeit und der Römischen Kaiserzeit (nach Martens 2007, S. 88 Fig. 1 - Ausschnitt).

Lange bekannt sind die Ringwallanlagen Heidenschanze und Heidenstadt in der Nähe von Sievern, Ldkr. Cuxhaven, von denen aber kaum aufschlussreiche Befunde vorliegen, was die mögliche Innenbebauung angeht. Die Heidenschanze ${ }^{31}$ besteht aus einem 2 ha großen umwallten Kernwerk und einer äußeren Umwallung, die 10 ha einschließt. Die mehrfach erneuerte Holz-Erde-Konstruktion der Befestigung war von der Mitte des 1. Jahrhundert v. Chr. bis an das Ende des 1. Jahrhundert n. Chr. in Funktion. Die erste Phase des 1,4 km langen Außenwalls war durch einen Zaun mit davor- und dahinter liegendem Graben nur recht schwach befestigt und datiert in die Jahrzehnte kurz vor oder um Chr. Geb. Die jüngere Phase ist ein 1,5 m hoher Wall, in den dann die Palisade aus mächtigen Spaltbohlen aus Eichenholz eingesetzt worden ist, insgesamt sind bei 1,4 km Länge 4200 solcher Bohlen verbaut worden, $79 \mathrm{n}$. Chr.

28 Müller 2007, S. 266 Abb. 1.

29 Mildenberger 1978.

30 Martens 2007, S. 88 Fig. 1.

31 Schmid 1999a; Schön 2000. 
dendrodatiert. Über die Innenbebauung ist aber (noch) wenig bekannt, anscheinend konzentrierten sich die Bauten hinter dem inneren Wall. ${ }^{32}$

In dieselbe Zeit gehört - so nahm man früher an - die in nur $800 \mathrm{~m}$ Entfernung gelegene Befestigung Heidenstadt, ${ }^{33}$ ein Ringwall von 220x180 m Durchmesser, wiederum ein Wall aus einer Holz-Erde-Konstruktion samt vorgelagerter Palisade und einem Graben. Neue Ausgrabungen im Innenraum und am Wall und den Toren ergeben aber andere Datierungshinweise. Danach gehört die Befestigung erst in das 4. und 5. Jahrhundert, und die Keramikfunde am Ort aus der Römischen Kaiserzeit kommen aus einer älteren, nicht befestigten Siedlung. ${ }^{34}$ Jüngst sind einige weitere Anlagen südlich von Cuxhaven bei Prospektionen entdeckt worden. ${ }^{35}$

Verglichen wird die befestigte Anlage Heidenschanze seit langem mit Befunden in den Niederlanden, Provinz Drenthe, den nahe beieinander liegenden umwallten Gehöften oder Speicherplätzen Zeijen I und II, Vries und Rhee, die jeweils sehr unterschiedliche Grundrisse und mehrere Ausbauphasen aufweisen. Die ersten Phasen gehören in die frühe Römische Kaiserzeit. Sie sind wesentlich kleiner als die beachtliche Anlage der Heidenschanze, entsprechen damit eher der üblichen Fläche umzäunter Gehöfte dieser Epoche, sie sind so klein, „daß sie einem massiven Angriff wohl kaum standgehalten hätten“. ${ }^{36}$

In Jütland sind inzwischen einige befestigte Siedlungen ausgegraben worden, die in die vorrömische Eisenzeit bis in die Zeit um Chr. Geb. datiert werden. Oftmals sind die Umwehrungen nur Gräben und schlichte Palisaden, es gibt aber auch stärker befestigte Anlagen.

$\mathrm{Zu}$ den ältesten bekannten Befestigungen gehört Borremose in Himmerland, Jütland, ${ }^{37}$ geschützt in einer Niederung gelegen mit einem starken Wall und breitem Graben, 150 m Nord-Süd und 80-100 m Ost-West messend (Abb. 6). Ein steingepflasterter Weg führt ins Innere, wo Grundrisse von 33 Langhäusern ergraben sind (Grabungen 1929-1945, in den 1960er Jahre und wieder 1989-1996), die jedoch nicht gleichzeitig gestanden haben. Die Siedlung wurde im 4. Jahrhundert v. Chr. gegründet und bestand bis ins 2. Jahrhundert v. Chr., also rund drei Jahrhunderte. Wieviele Gebäude gleichzeitig gestanden haben, ist leider aufgrund des unscheinbaren Fundmaterials nicht sicher zu beschreiben; jedoch heben sich in den verschiedenen Phasen jeweils Baukomplexe aus mehreren, meist zwei oder drei Häusern aufgrund ihrer Größe und Lage heraus, die als „Herrenhöfe“ gedeutet werden, als Sitz des Siedlungsgründers und reichsten Familienverbandes.

32 Aufderhaar et al. 2009 (2011) S. 2003-2006 (Iris Aufderhaar).

33 Schmid 1999b.

34 Aufderhaar et al. 2009 (2011) S.194-202 (Matthias D. Schön)

35 Mündliche Mitteilungen bei der Tagung 2012 in Göttingen.

36 Waterbolk 2007, S. 496.

37 Martens 2010; 2007, S. 89-93. 


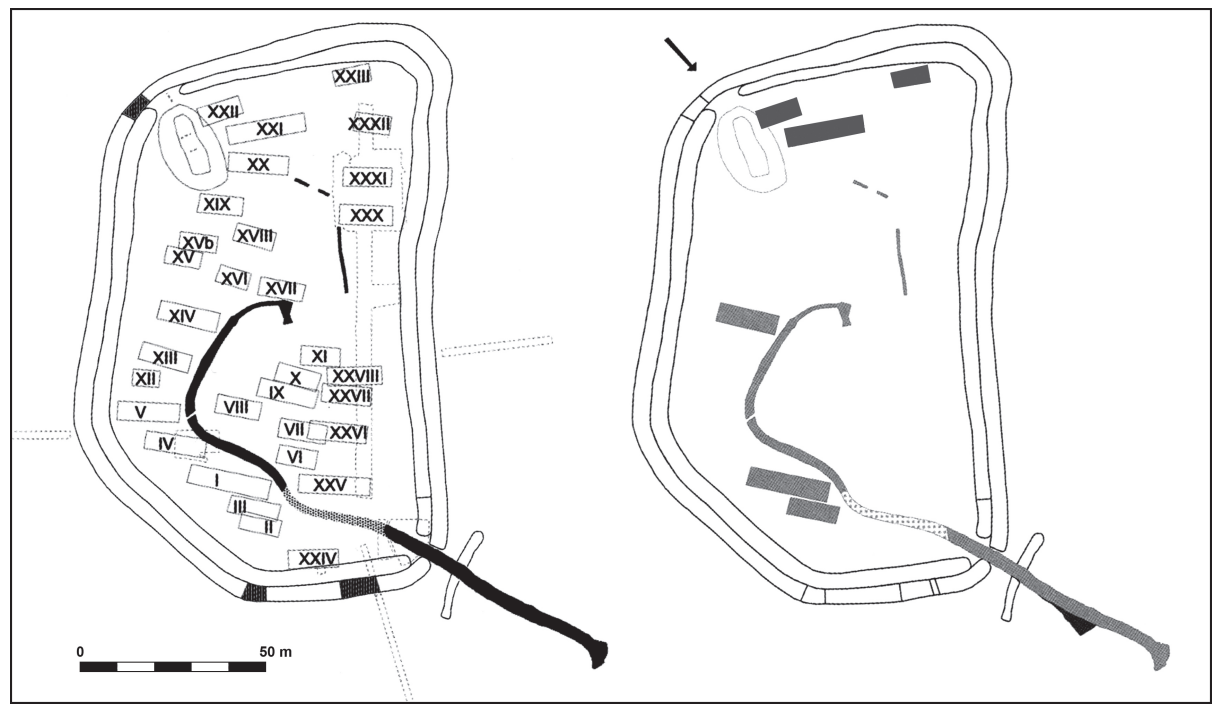

Abb. 6: Befestigte Siedlung von Borremose, Nordjütland. 1 Grundriß mit sämtlichen, aber nicht gleichzeitig stehenden Häusern, 2 Lage des Gründergehöftes (dunkelgrau) und Langhäuser mit ausgeprägtem Wohnteil (nach Martens 2010, S. 186 Fig. 6 und S. 191 Fig. 16).

Etwas jünger, aber ähnlich in der Lage, hier auf einem leichten Höhenzug, und vergleichbarer Struktur, ist die befestigte Siedlung von Lyngsmose im mittleren Westjütland nahe Ringkøbing (ausgegraben 2000-2002), 90 x 60 m messend (Abb. 7). ${ }^{38}$ Mit dem Hinweis darauf, dass die Siedlungen der vorrömischen Eisenzeit in Dänemark dicht beieinander, oft nur $2 \mathrm{~km}$ von einander entfernt lagen, wird diese stark befestigte mehrphasige Siedlung innerhalb der Siedlungsstreuung eingeordnet. Sie ist mit einem breiten und tiefen Graben, in denen angespitzte Hölzer eingesetzt waren, gesichert. Zahlreiche Wohn-Stall-Häuser sind nachgewiesen, also Gebäude für Landwirtschaft und Viehhaltung (mit Boxen für die Rinder). Von denen sind im südlichen Bereich in der älteren Phase um 100 v. Chr. mit sechs Gebäuden zwei bis $13 \mathrm{~m}$ lang Häuser durch einen inneren Zaun zusammengefasst. In der jüngeren Phase um Chr. Geb. standen 15 größere und zwei kleinere Häuser in der Befestigung. Die Ausgräber schätzen insgesamt 120 bis 150 Bewohner.

In diese späte Phase der vorrömischen Eisenzeit bis um Chr. Geb. gehört auch die schon länger bekannte ebenfalls mehrphasige Siedlung Hodde in Westjütland. ${ }^{39}$ Hier ist eine größere Anzahl von dreischiffigen Wohn-Stall-Häusern (mit Vieh-Boxen) insgesamt von einer Palisade eingehegt, und innerhalb der Ansiedlung sind wiederum einige Hauskomplexe mit einem zusätzlichen Zaun umschlossen. Ein Hofkomplex im Norden besteht aus dem größten Haus der Siedlung, das zudem besonders

38 Eriksen / Rindel 2005; auch Rindel 2001.

39 Hvass 1985; 1997, S. 392 f. mit Fig. 1-3; Martens 2010, S. 182 Fig. 1; vgl. Steuer 2007, S. 347 f. mit Abb. 5. 
stark befestigt ist, und einem zugehörigen kleineren Gebäude ohne Stallteil, ebenfalls extra eingezäunt. Aufgrund des engen Tores in der Umzäunung, noch unterteilt durch einen starken Pfosten, wird dieses Haus als Kultgebäude gedeutet. ${ }^{40}$ Die größte Anzahl von Häusern wird in die Zeit um Chr. Geburt datiert. Der besondere Hofkomplex, der zudem über einige Generationen am Ort bestand und mehrfach erneuert wurde, wird als Häuptlings-Farm gedeutet.
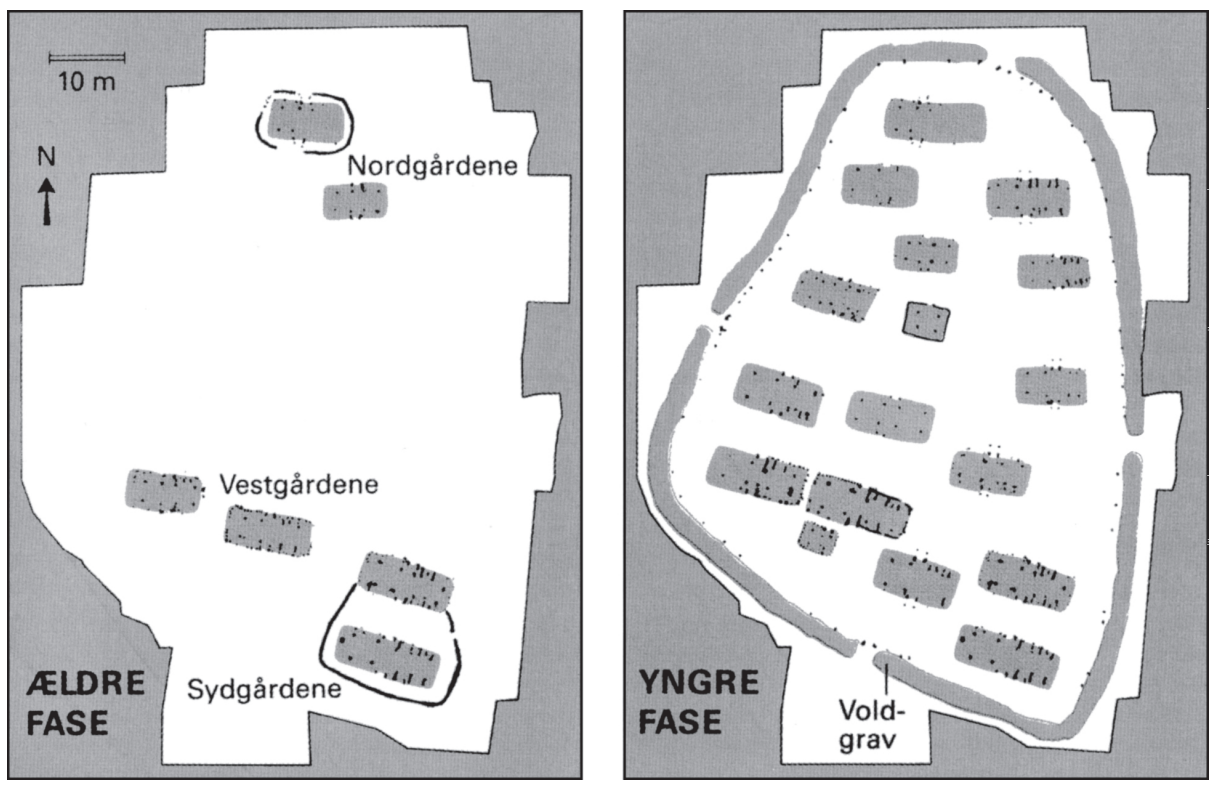

Abb. 7: Befestigte Siedlung von Lyngsmose, Westjütland. 1 Ältere Phase, links, 2 Jüngere Phase, rechts (nach Martens 2007, S. 93 Fig. 5).

Die zuerst in der Forschung als Herrengehöft angesprochenen Baustrukturen wurden von 1954 bis 1963 von Werner Haarnagel auf der Feddersen Wierde ausgegraben. ${ }^{41}$ In der Wesermarsch wurde eine Flachsiedlung im 1. Jahrhundert v. Chr. gegründet und bis ins 1. Jahrhundert $\mathrm{n}$. Chr. in vier Phasen ausgebaut mit bis zu 11 Wirtschaftseinheiten. (Siedlungshorizont [SH] - SH 1a: 5 Familien Wohn-Stall-Haus mit Speicher; SH 1b: 8 Betriebe mit 20 m langen Häusern; SH 1c: 8 Betriebe, die leicht verlagert sind gegenüber den älteren Strukturen; SH 1d: nun schon 11 Wirtschaftsbetriebe, unter denen sich ein Gehöft heraushebt: es besteht aus 2 kleinen und 2 großen Wirtschaftsbetrieben, ein Haus von 28 m Länge und eine Mehrbetriebseinheit). Erst in der Phase SH 2 errichtete man die ovale Anordnung einer größer werdenden Wurt. Innerhalb von 250 Jahren erfolgte ein Anstieg der Zahl der Gehöfte von 5 auf 26. Im Südwesten

40 Martens 2010, S. 182 mit Fig. 1.

41 Schmid 1994; Schmid 2010; Burmeister / Wendoski-Schünemann 2010. 
der Wurt entwickelte sich ein besonders großer Gehöftkomplex, der als Herrenhof bezeichnet wird. Im Siedlungshorizont 4 in der 2. Hälfte des 2. Jahrhunderts n. Chr. ist diese Anlage mit einer Palisade befestigt, die mehrere Wohn-Stall-Häuser, Speicher und Werkplätze, aber auch - das ist wichtig - zwei große dreischiffige Gebäude umschließt, in denen kein Vieh aufgestellt werden konnte, sog. Versammlungshäuser. Dieser Großhof war schon im Siedlungshorizont 1d im frühen 1. Jahrhundert zu erkennen und entwickelte sich also an derselben Stelle über mehrere Jahrhunderte und einige Generationen immer weiter. Über seine Funktion entspann sich in den letzten Jahren eine Diskussion, auch verbunden mit einer neuen Auswertung der alten Grabungsbefunde. Doch bleibt es bei der besonderen Struktur dieses Anwesens. ${ }^{42}$

Der Übergang vom Zaun zur stärkeren Palisadenbefestigung ist natürlich fließend; oft sind auch nur die mehr oder weniger breiten Gräben bei den Ausgrabungen erfasst worden. Als Beispiel bringe ich die Siedlung Oss-Almstein aus den südlichen Niederlanden links des Rheins. Hier sind die zweischiffigen Wohn-Stall-Häuser fast immer an derselben Stelle wieder errichtet worden, die Siedlung wanderte also nicht, und der breite Graben schloss mehrere Häuser ein. ${ }^{43}$

In diesem Zusammenhang sei auch noch die Siedlung Priorsløkk $e^{44}$ beim Horsens Fjord in Jütland genannt, weil hier ein besonders komplizierter Befund vorzuliegen scheint. Ein Befestigungsgraben schließt die gesamte Siedlung mit zahlreichen Wohn-Stall-Häusern bzw. Gehöften ein, datiert in die ältere Römische Kaiserzeit. Bei Ausgrabungen 1980-1983 wurden auf einem Areal von 150 zu 75 m 47 Häuser unterschiedlicher Größe, zweiphasig auf einer Insel in der sumpfigen Niederung erfasst. Die erste Hauptphase bestand aus 14 bis zu 12 m langen Häusern, die zweite Phase aus drei 15-16 m langen Häusern. Die Siedlung bestand insgesamt bis höchstens 200 n. Chr. und wurde kriegerisch zerstört. Ein $3 \mathrm{~m}$ breiter und bald $2 \mathrm{~m}$ tiefer Befestigungsgraben mit einer Palisade dahinter wurde in der Spätphase, erst kurz vor der Zerstörung der Siedlung errichtet. Die Ausgräber meinen, dass dieses Dorf somit nicht als befestigte Siedlung zu betrachten sei; denn die Anzahl der verbauten Palisadenpfosten entspräche genau der Anzahl aller Hauspfosten. Erst durch die Zerstörung des Dorfes war der Bau der Befestigung möglich. Weil die Siedlung inmitten eines größeren Territoriums lag, über das ein Häuptling geherrscht habe, hatte er als Kriegsfürst die Möglichkeit zu befehlen, das Dorf zu zerstören, um eine sichere Befestigung zu bauen. Experimente haben gezeigt, dass nur 40 Männer in einer Woche Graben und Palisade haben bauen können. Der komplexe Befund spiegelt einen innergermanischen Krieg, in einer Zeit, als auch im Moor von Illerup das erste Kriegsbeuteopfer niedergelegt worden ist. Erinnert sei an die flüchtigen Befestigungen Heidenschanze und Heidenstadt an der südlichen Nordseeküste, die auf ähnliche Weise entstanden sein können.

42 Schmid 2010; Burmeister / Wendoski-Schünemann 2010.

43 Jansen / Fokkens 2010, S. 141 f. mit Fig. 8.

44 Kaul 1999, S. 60 ff. mit Fig. 9; 2003. 
Überblickt man die Befunde, so kann zusammengefasst werden, dass vom 4. Jahrhundert v. Chr. bis ins 2. Jahrhundert n. Chr. immer wieder befestigte Siedlungen, oft mit einem größeren, besonders geschützten Hof archäologisch nachgewiesen sind. Vom Areal her ist die Einwohnerzahl sicherlich nicht gering gewesen. Zum belagerten Hof des Segestes bieten diese Befunde reale Beispiele als Vergleich an.

Es muß aber angemerkt werden, dass der Kenntnisstand zu „germanischen“ Befestigungen der Römischen Kaiserzeit nördlich des Mittelgebirgsrandes immer noch völlig unbefriedigend ist. Seit der Zusammenstellung von Gerhard Mildenberger von $1978^{45}$ hat sich das Bild nicht wesentlich geändert; es gibt viele fragliche und unklare Befunde. Mildenberger hatte die Befestigung „Schwedenschanze“ bei Isingerode an der Oder erwähnt, ein Ringwall von 180 auf 140 m Abmessung; erneut wurde dort ausgegraben und eine bronzezeitliche Befestigung nachgewiesen. Dabei zeigte sich außerdem, daß der jungbronzezeitliche innere Wehrgraben erneut ausgehoben und auf dem alten Wall eine Holzplankenmauer mit mächtigen Stützpfosten errichtet worden war. Diese Erneuerung wird in die Zeit zwischen 12 v. und 16 n. Chr. datiert, ${ }^{46}$ in die Zeit der römischen Angriffskriege. Die Deutung als Befestigung liegt nahe, aber weitere Aussagen zum Inneren der Anlage sind bisher nicht möglich. Doch zeigt dieses Beispiel, dass mit vielerlei Überraschungen gerechnet werden kann, wenn Ausgrabungen intensiver fortgesetzt und tatsächlich alle Phasen einer Befestigung auch erkannt werden.

Schließlich sei noch auf einige Wallanlagen auf den Nordfriesischen Inseln hingewiesen, die zwar archäologisch untersucht sind, aber wenig Aufschlüsse über eine ehemalige Innenbebauung bieten. Es handelt sich um die Archsumburg und die Tinnumburg auf Sylt, die Lembecksburg auf Föhr und die Anlage Trælbanken in NordSchleswig. Sie werden als kultische Zentren oder lokale Opferstätten der umliegenden Bevölkerung gedeutet, die auch wegen der geringen Wallhöhe der Ausbauphase während der Römischen Kaiserzeit keine fortifikatorische Funktion gehabt haben können. ${ }^{47}$ Die heutige Wallhöhe der Tinnumburg von über $5 \mathrm{~m}$ entstammt erst der Wikingerzeit, aus der auch Gebäude im Innenraum nachgewiesen sind. Sie werden aber auch anders interpretiert, nicht als befestigte Herrenhöfe oder Opferstellen, sondern als „große kollektive Verteidigungsanlagen“, ${ }^{48}$ politisch verglichen mit den Landwehren wie dem $12 \mathrm{~km}$ langen Olgerdiget, der jetzt in seiner ersten Phase um 50 n. Chr. datiert und von der Anlage $Æ$ Vold ab 125 n. Chr. abgelöst wird, gelegen in Jütland.

45 Mildenberger 1978, Karte 3 und 4.

46 Steinmetz 2013, S. 57.

47 Harck 1990; Brieske 2005, S. 614.

48 Ethelberg 2009, S. 174 f.; auch Ethelberg 2007; Christensen 2006b. 


\section{Großhof und Halle - Hierarchie in der Siedlung}

Wie bei den befestigten Siedlungen, für die Beispiele über Jahrhunderte zu verfolgen sind, bieten auch die zahlreichen ausgegrabenen Siedlungsplätze jeweils sehr individuelle Befunde, kaum Regelerscheinungen, was daran liegen wird, dass noch immer nur eine kleine Auswahl ehemals existierender Ansiedlungen erschlossen worden ist, wobei nicht sicher ist, ab wann ein repräsentativer, statistisch ausreichender Querschnitt für die auswertende Forschung vorliegt. Regelhaft $\mathrm{zu}$ beobachten ist aber die Vielphasigkeit aller Plätze; denn Häuser und die gesamten Siedlungen werden in Generationsabständen von 20 bis 30 Jahren erneuert, bei einer Wurt am selben Platz durch Aufhöhung, auf festem Land durch Verlagerung der gesamten Siedlung. Dies geschah sicherlich mit Blick auf die Ackerflur, über die wir aber nur recht wenig wissen. Andererseits erstrecken sich - wenn genügend große Areale freigelegt werden - diese Siedlungen bei unterschiedlich erfasstem Beginn über einen langen Zeitraum von Jahrhunderten, gar einem Jahrtausend.

Mit dem Blick auf die Epoche der Germanenkriege ist festzustellen, dass diese militärischen Ereignisse keinen Einschnitt im Besiedlungsmuster in der Germania ausgelöst haben; vielmehr steht hinter der Geschichte einer dörflichen Ansiedlung ein jeweils individuelles Schicksal. Es macht aber Mühe, gerade die Phase der ersten Jahrzehnte $n$. Chr. herauszuarbeiten und vorzustellen, da oftmals die wandernden Siedlungen in dieser Zeit erstmal zu erfassen sind.

Zwei charakteristische Elemente seien beschrieben: Zum einen kann für fast jede Siedlung - wenn ausreichend ausgegraben - eine Hierarchie der Gehöfte, was Größe, Viehstapel und Zahl der Gebäude pro Gehöft angeht, festgestellt werden. Ob man beim größten Gehöft von Dorfherrschaft sprechen darf oder besser nur unterschiedliche Ränge innerhalb einer Siedlung erkennen kann, soll hier nicht diskutiert werden. Zum anderen gibt es in manchen dieser Großhöfe ein hallenartiges Gebäude, das weder zur Unterbringung von Vieh, noch zum Wohnen einer Familie gedient hat, sondern als Versammlungs-, Fest- und/oder Kulthalle gedacht war.

Die lange Existenz, bei regelmäßiger Verlagerung, einer Siedlung veranschaulicht immer noch am besten das Dorf Vorbasse, Vejle Amt, in Jütland (Abb. 8) ${ }^{49}$ In den Jahren 1974 bis 1987 wurden 23 ha Fläche freigelegt und ein über 1100 Jahre lang bestehendes wanderndes Dorf erschlossen. Um Chr. Geb. setzten im ausgegrabenen Teil innerhalb eines $700 \times 100$ m großen Gebietes zeitgleich drei Siedlungen ein, in der Mitte ein Dorf aus mehreren Höfen, links ein eingezäuntes Gehöft mit einem Haupthaus und zwei Nebengebäuden, also mit komplexer Gehöftstruktur. Die nächste Verlegung aller drei Siedlungen folgte in der Zeit von 100 bis 200 n. Chr., was sich bis über die Wikingerzeit hinaus fortsetzte.

49 Hvass 2006. 


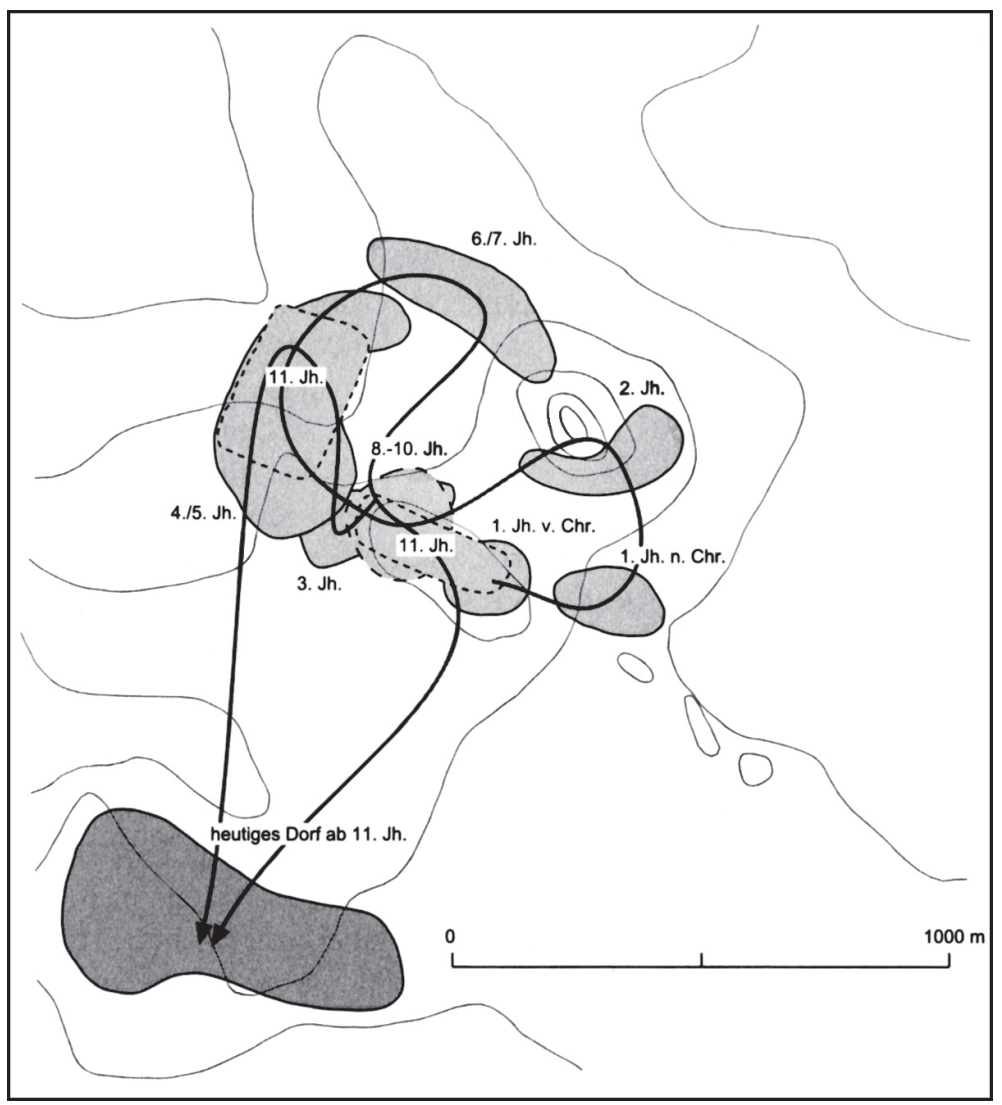

Abb. 8: Vorbasse, Mitteljütland (nach Brather 2006, S. 74 Abb. 10 - verändert nach Hvass 2006).

Die wandernde Siedlung von 8 ha Größe bei Nørre Snede, Vejle Amt, wurde großflächig von 1979 bis 1986 erforscht..$^{50}$ Sie bestand aus Dutzenden von Gehöften (166 Langhäuser und 132 Kleinhäuser), die sich linear von Südosten nach Nordwesten verlagern. Die älteste Phase im ausgegrabenen Bereich gehört ins 1. Jahrhundert n. Chr., und die Siedlung existierte bis ins frühe 8. Jahrhundert, mit einer fast gleichbleibenden strengen rechteckigen Parzellenstruktur, ablesbar an den Zaunverläufen. ${ }^{51}$

50 Hvass 1997, S. 406-409 mit Fig. 18-21 mit der Phasenabfolge im Gesamtplan der Siedlung; Holst 2010; Egeberg Hansen / Holst 2002.

51 Holst 2010, S. 162 Fig. 1: Lage in Mitteljütland nicht fern von Vorbasse, S. 163 Fig. 2 Gesamtplan der Ausgrabung, S. 165 Fig. 4 Zeittafel zu den Phasen. 
Weitere Beispiele aus Jütland seien noch genannt: Galsted ${ }^{52}$ aus dem 1. Jahrhundert n. Chr., wo sich das Gehöft des „Großbauern“ deutlich heraushebt, ${ }^{53}$ und Vestergård ${ }^{54}$ oder Garslev, ${ }^{55}$ außerdem Osterrönfeld, Kr. Rensburg-Eckernförde. ${ }^{56}$ Für diese Siedlung beschreibt Per Ethelberg einen Haustyp und kartiert dessen Verbreitung im westlichen Jütland; und diese Karte zeigt wiederum, wie dicht die seinerzeitigen Siedlungen beieinander gelegen haben. ${ }^{57}$ In Askov bei Vejen im mittleren Jütland wurde eine Siedlung der Zeit von 100 v. bis 50 n. Chr. ausgegraben, mit mehr als 25 Gehöften, die im Grabungsareal seinerzeit gleichzeitig gestanden haben. ${ }^{58}$ Ein Reihendorf aus der frühen Römischen Kaiserzeit wurde bei „Klosterbakken B“ bei Rødekro im südlichen Jütland großflächig ausgegraben. Der Platz gehört zu weiteren unmittelbar benachbarten Siedlunsgarealen wie „Klosterbakken D“ als Vorgänger aus der späten vorrömischen Eisenzeit und „Klosterbakken C“ als Nachfolger aus der Römischen Kaiserzeit. Zäune und beachtliche Langhäuser bezeugen Großgehöfte. ${ }^{59}$

Gekoppelt mit einer umfangreichen Eisenverhüttung auch schon in der Römischen Kaiserzeit sind beispielhaft die Siedlungen Drengsted und Snorup (Abb. 9). ${ }^{60}$ Über die große Bedeutung der dörflichen Eisenproduktion in der Germania als wichtiger Wirtschaftsfaktor zumindest für die Eigenversorgung, teils aber sicherlich auch mit einer Überproduktion, seit der Zeit um Chr. Geb. informiert Hauke Jöns, u. a. an der von ihm ausgegrabenen Siedlung Joldelund. ${ }^{61}$

Südlich der Nordseeküste wurde eine komplette Siedlungskammer im Ldkr. Cuxhaven archäologisch untersucht, mit Flögeln ${ }^{62}$, einem Dorf der Römischen Kaiserzeit und der Völkerwanderungszeit in der Mitte. Die Grabungen dauerten von 1971 bis 1986 und darüber hinaus; über 150 Langhäuser und ebenso über 150 Grubenhäuser des 1. Jahrhunderts v. bis 6. Jahrhundert n. Chr. wurden freigelegt. Im Grabungsareal wird der Siedlungsbeginn zur vorrömischen Eisenzeit und frühen Römischen Kaiserzeit erfasst. Es handelt sich um eine Streusiedlung im Bereich der Ackerflächen, der sog. celtic fields von 100 ha Umfang. Innerhalb und am Rande verteilt liegen die Einzelhöfe

52 Ethelberg 1995; 2001; 2007; 2009, S. 173 Abb. 4 in Farbe.

53 Ethelberg 2001: S. 60 Abb. 3 Galsted Dorf A 1. Jh. n. Chr., jüngste Siedlungsphase ältere Römische Kaiserzeit 2. Jh. n. Chr., S. 61 Abb. 3 A und B: älteste Siedlungsphase der älteren Römischen Kaiserzeit (1. Jh.) - weitere Siedlungen S. 64 ff. mit Abb. 8 Osterrönfeld, S. 67 f. Vestergard , S. 68 mit Abb. 13 Hjøhøj, S. 70 f. mit Abb. 15 sowie 16 Johannesminde und schließlich S. 72 mit Abb. 17 Skovminde.

54 Christensen 2006a.

55 Hvass 1997, S. 395 Fig. 5 Großhof des 1./2. Jh. n. Chr.

56 Ethelberg 2009, 174 Abb. 5 Plan der Siedlungsgrabung Osterrönfeld.

57 Ethelberg 2009, S. 176 Abb. 7: Verbreitung der Häuser vom Typ Osterrönfeld; eine weitere Verdichtung der Verbreitung dieses Haustyps in wenigen Jahren: Ethelberg / Kruse 2013, S. 104 Abb. 1.

58 Eisenschmidt 2006, S. 60 mit Abb. 2.

59 Lütjens 2008.

60 Nørbach 1999, S. 243 Fig. 6 und 7; zu Snorup besonders Nørbach 2005, Plan S. 179 Abb. 24. Häufung der Eisenverhüttungsöfen.

61 Jöns 1999; 2007; 2010.

62 Zimmermann 1995a; 1995b. Gesamtplan jetzt z.B. bei Dübner 2013, S. 226 Abb. 1. 
mit Wohn-Stall-Haus, Grubenhaus und ebenerdigem Nebengebäude. In der Grabungsfläche sind sechs Gehöfte angeschnitten. Die späteren Phasen ab dem 2. Jahrhundert sehen dann ein geschlossenes Dorf mit umzäunten sog. Mehrbetriebseinheiten - d.h. mit mehreren Wohn-Stall-Häusern innerhalb eines Gehöftplatzes -, die verschieden umfangreich sind und ebenfalls Rangunterschiede erkennen lassen. ${ }^{63}$ Ein weiteres Dorf auf der Geest ähnlich wie Flögeln ist bei Loxstedt ausgegraben worden, eine Wandersiedlung vom 1. Jahrhundert v. bis ins 3. Jahrhundert n. Chr. ${ }^{64}$

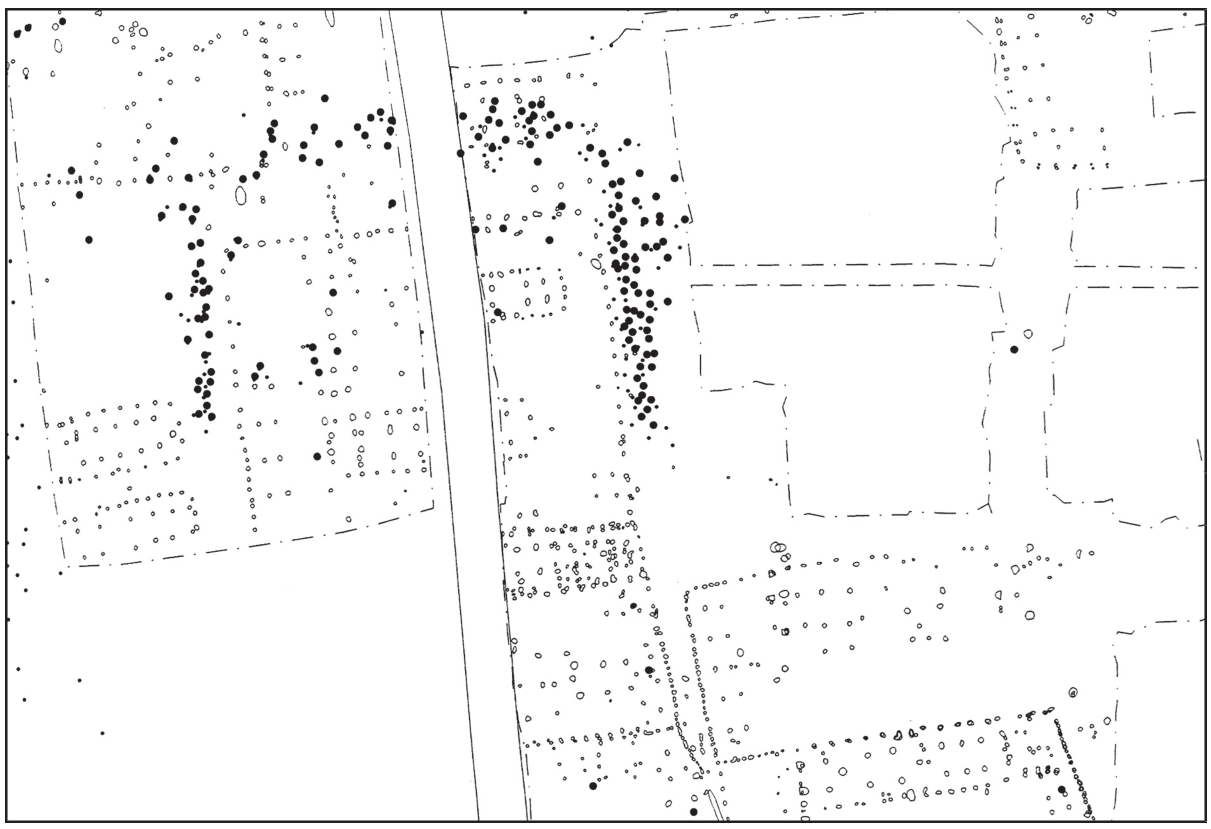

Abb. 9: Snorup, Ldkr. Ribe, Jütland. Ausschnitt aus dem Siedlungsplan (vollständig bei Nørbach 2005, S.179 Abb. 24). Außen am Zaun eines Gehöftes gereihte Eisenerzverhüttungsöfen (nach Nørbach 1999, S. 243 Fig. 7 - verändert).

Inzwischen gibt es weitere derartige Siedlungen auch in benachbarten norddeutschen Arealen, z.B. Groß Meckelsen, Ldkr. Rotenburg (Wümme), mit mehr als 100 Langhäusern, über 100 Grubenhäusern und 300 Rennfeueröfen. ${ }^{65}$ Schon vor langer Zeit wurde 1958 als erster Einstieg, leider nur unvollkommen publiziert, eine Dorfsiedlung bei Gristede im Ammerland ausgegraben, etwa 4 ha. Die Siedlung beginnt in der vorrömischen Eisenzeit und reicht bis in die Zeit um $500 .{ }^{66}$

63 Zimmermann 1995b, S. 262 Abb. 8: Streusiedlung des 1. Jahrhunderts n. Chr. - Neue Auswertung der Gehöftstrukturen Dübner 2013, S. 228 f. Abb. 2, 1-13: Phasenabfolge von 1. bis 4. Jh. n. Chr.

64 Zimmermann 2001.

65 Tempel 2003; 2004; Bock 2013. Ausgrabungen von 1987 bis 2002 und 2011.

66 Kauffmann 1999. 
Aus Jütland und den Niederlanden ließe sich die Zahl der vergleichbaren Beispiele noch deutlich vergrößern. Ebenso gibt es häufig den Beleg für ein innerhalb der dörflichen Siedlung herausgehobenes Gehöft, das meist für sich noch einmal mit Palisaden umgeben war. ${ }^{67}$ Auch in anderen Gebieten, dem östlichen Deutschland sind inzwischen großflächig Siedlungen der frühen römischen Kaiserzeit erforscht worden, z.B. Herzsprung in der Uckermark. ${ }^{68}$ Hier sind Großhöfe freigelegt worden, die den Vergleich mit norddeutschen und dänischen Befunden erlauben.

Es gibt noch spezielle Typen von Siedlungen, weil dort die Häuser anders gebaut worden sind, mit breiten Sodenmauern, so bei Archsum auf Sylt ${ }^{69}$ oder in Vestervig, Jütland. ${ }^{70}$

Die Siedlung Vestervig besteht von 100 v. Chr. bis 450 n. Chr. aus sieben Phasen.

\subsection{Hallen $^{71}$}

Ein Herrenhof ist durch mehrere Elemente gekennzeichnet, durch die Größe der Wirtschaftseinheit Wohn-Stall-Haus, durch ein weiteres Hallengebäude, das von der Größe her über die Hofbewohner hinaus als Versammlungsraum diente, in dem Feste gefeiert und Kulthandlungen vollzogen werden konnten.

Über das Problem der Bezeichnung kann hier nicht weiter diskutiert werden, nämlich was ein Herrenhof war, ein Häuptlingshof, ein Herrschaftssitz, ein Adelshof, in anderer Sprache a magnate's farm, a chieftain's farm, a stormands gårde. ${ }^{72}$ Eindeutig bleibt jedoch der Rangunterschied zwischen einem solchen Herrenhof und den übrigen Gehöften einer dörflichen Siedlung.

Unstreitig sind reine Hallenbauten auf der Feddersen Wierde seit dem Siedlungshorizont 3, zu Beginn des 2. Jahrhunderts n. Chr., aber vielleicht schon angedeutet ein Vorläufer im SH 1d bald nach der Zeitenwende zu beobachten. ${ }^{73}$ Für die Folgezeit sind Hallen-Gebäude immer wieder in jeweils einem der Gehöfte einer dörflichen Siedlung nachzuweisen, die im Verlauf der Jahrhunderte auch immer größer werden, bis hin zu den 90 m langen Hallen in Borg auf den Lofoten aus der Wikingerzeit. ${ }^{74}$

Auch ist der Zusammenhang von Halle und Kultbau nach den jüngsten Fund neu zu überdenken. Da Jes Martens für Haus I von Borremose aufgrund besonderer Beobachtungen (große Vorratsgefäße bei der Herdstelle als Versorgungs- und Speicherbe-

67 Martens 2010, S. 183 mit Fig. 2 (Omgård-Sig und Grønberg skole, West-Jütland); Hvass 1997, S. 394 Fig. 4, S. 395 Fig. 5 (Gårslev bei Vejle).

68 Schuster 2004.

69 Kossack 1997; Lütjens 2006; 2008.

70 Kaul 1999; 2006.

71 Herschend 1993; 1999; Løken 2001.

72 Martens 2010, S. 182 und Burmeister / Wendowski-Schüneman 2010, S. 45 ff.

73 Burmeister / Wendowski-Schünemann 2010, S. 49; Schmid 2010.

74 Magnus 2001; Munch 2003. 
hälter) eine Funktion als Gast- und Hallenhaus vermutet, käme man in die jüngere Phase der Befestigung, also schon ins 2. Jahrhundert v. Chr. ${ }^{75}$

Trond Løken bietet 2001 ebenfalls schon für die ältere Römische Kaiserzeit aus Norwegen (Rogaland) Beispiele für reine Hallengebäude an, ${ }^{76}$ ergänzt durch Beispiele aus Dänemark, von Hodde bis Nørre Snede in Jütland und Helgö in Mittelschweden. Er geht von Norwegen aus, wo er für die Zeit um Chr. Geb. eine stratifizierte Gesellschaft annimmt, weshalb dort auf dem Haupthof einer Siedlung auch ein „öffentlicher Raum“, eine Halle nötig war, in dem Festlichkeiten und Kulthandlungen hätten durchgeführt sowie Leitungsfunktionen hätten übernommen werden können. Anfangs könne u.U. auch schon nur ein Teil des Hauses dazu gedient haben, nämlich im Plan erkennbar, wenn die Mitte des Hauses weit auseinander stehende Pfosten aufweist und es also einen größeren freien Raum gab. Nachfolgend wurden in der späten Römischen Kaiserzeit separate Hallengebäude errichtet. Seine Hypothese ist gar, dass die Halle als öffentlicher Raum vor dem Ende der vorrömischen Eisenzeit aufgekommen sein muss, 300 bis 400 Jahre früher als bisher angenommen, früher als im Borremose des 2. Jahrhunderts v. Chr. oder mehr noch vor der Feddersen Wierde um Chr. Geb. bzw. dem 1. Jahrhundert n. Chr.

Nicht übersehen werden sollte dabei aber außerdem, dass mehrfach langrechteckige reine Pfostenbauten parallel zum Gehöftzaun auch als Ställe gedeutet werden.

\subsection{Kultbau}

Für die Siedlung mit Großhof von Hodde, in dessen Umfeld auch wertvollere Keramik und anderes qualitätsvolleres Material gefunden worden ist als in der Siedlung sonst, wird ein Haus als Kultgebäude interpretiert, was die hervorgehobene Stellung des „Herrenhofes“ unterstreicht. Das doppelt eingezäunte, relativ kleine Gebäude auf dem Herrenhof hat einen besonderen, durch einen schweren Pfosten noch einmal unterteilten Eingang, den nur Menschen einzeln passieren konnten. ${ }^{77}$

Im schwedischen Uppåkra bei Lund ist ebenfalls ein solches Vier-PfostenGebäude entdeckt und ausgegraben worden, das etwa vom letzten Jahrhundert vor Chr. Geb. bis in die Wikingerzeit an derselben Stelle immer wieder neu errichtet worden ist. Für spätere Phasen ist über Waffendeponierungen und niedergelegte Goldblechfigürchen der religiöse Charakter gesichert, so dass er auch zu Beginn als Kultbau gedient haben wird. ${ }^{78}$

75 Martens 2010, S. 190 f. mit Fig. 13-14.

76 Løken 2001, S. 56 f. Fig. 2 c-d.

77 Martens 2010, S. 182 mit Fig. 1.

78 Larsson (Ed.) 2004; Hårdh 2006; Larsson 2013. 


\subsection{Römischer Einfluss?}

Im mittleren Jütland ist bei Tjørring ${ }^{79}$ seit 1993 ebenfalls großflächig auf 23 Hektar eine wandernde Siedlung ausgegraben worden (Abb. 10 und 11). Die Gehöfte decken eine Zeitspanne von 500 v. Chr. bis 200 n. Chr. ab. In regelmäßiger Abfolge heben sind auch Großhöfe heraus. Das rechteckige Gehöft des 1. Jahrhunderts n. Chr. mit den Abmessungen von ca. 60 auf 80 m und zahlreichen Gebäuden innerhalb der Umzäunung macht einen so sorgfältig vermessenen Eindruck, dass vermutet wurde, dahinter würde der römische Fuß von 29,6 cm stehen, was aber nicht der Fall ist. Vielmehr ergibt ein „prähistorisches“ eigenes Fußmaß von 16,5 bzw. 33 cm ebenfalls sinnvolle Abmessungen und Maßverhältnisse. Das regte aber trotzdem dazu an, dieses Gehöft mit römischen Villenanlagen im Rheinland zu vergleichen.

Gräber mit auffälligen Beigaben, darunter wertvoller Schmuck und römische Bronzegefäße der Phase B1a (30-40 n. Chr.), in unmittelbarer Nähe spiegeln Kontakte zur römischen Welt. Wie dieser Einfluss zustande gekommen sein kann, mag man diskutieren: Söldnerdienst in einer Auxilia wie die Krieger des Arminius oder der Bataver; oder bei der in den Res gestae des Augustus erwähnten Expedition zur Kimbrischen Halbinsel oder der Expedition des Tiberius im Jahr 5 n. Chr. an die Küsten Jütlands oder Handelskontakte.

Ein Blick in das Gebiet der Bataver westlich des Niederrheins zeigt aber durchaus ein anderes auffälliges Bild, nämlich die Beharrung in der Tradition germanischer Gehöftanlagen in manchen Landschaften zwischen Bereichen, in denen sich schon tatsächlich diese römische Villen-Kultur ausgebreitet hatte. ${ }^{80}$ Die Bataver blieben konservativ beim Wohn-Stall-Haus mit eingegrabenen Holzpfosten und Lehmwänden. Nur in einigen Fällen hatte man dem zweischiffigen Langhaus noch einen Säulenumgang, eine Portikus angefügt. Nico Roymans meint deshalb: „Diese Hybridformen illustrieren den hohen symbolischen Wert des einheimischen Wohnstallhauses innerhalb des batavischen Selbstwertgefühls, gleichzeitig sind die hinzugefügten Architekturelemente eine Aussage über die römische Identität und die damit verbundenen Absichten der Bewohner. Die Erbauer dieser romanisierten Bauernhäuser... müssen wir im Umfeld von ehemaligen Soldaten (und Offizieren) suchen, die über den entsprechenden Reichtum und Verbindungen zum Militär verfügten“ und weiter „Die Wohnstallhäuser und die Gräberfelder sind Symbole einer vorväterlichen Vergangenheit und gehören zum symbolischen Vokabular der Bataver bei der Verdeutlichung ihrer eigenen Identität“ .81

79 Møller-Jensen 2010.

80 Roymans 2009 mit Karte Abb. 5 auf S. 90.

81 Roymans 2009, S. 92 und S. 93. 


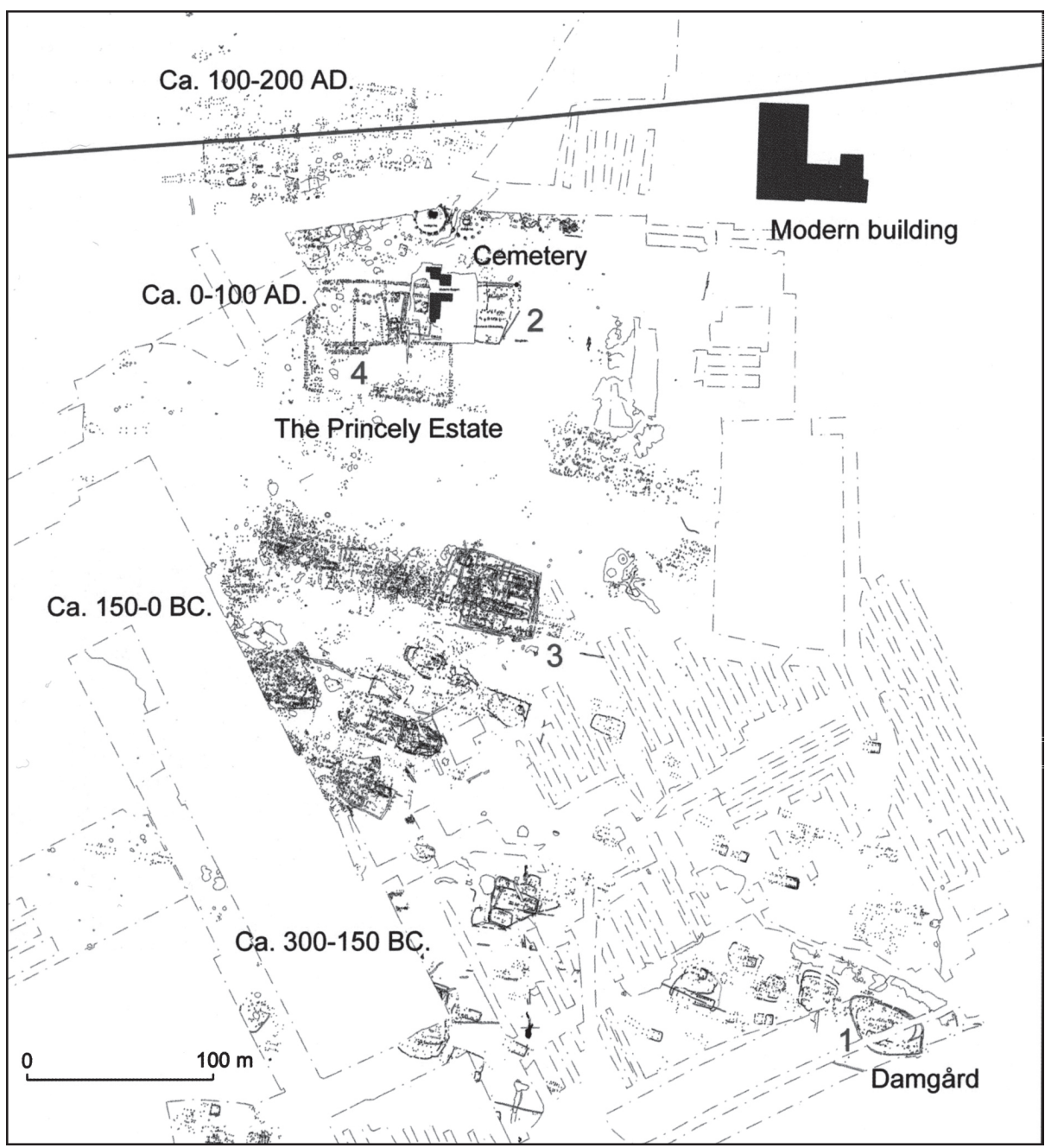

Abb. 10: Tjørring, Jütland. Phasen der Siedlung (nach Møller-Jensen 2010, S. 199 Fig. 2 - Ausschnitt).

Auf einige weitere Beispiele derartiger rechteckig eingezäunter Parzellen mit mehrfacher Bebauung in der Maaskant Region der südlichen Niederlande, die wie Herrenhöfe wirken, sei noch hingewiesen. ${ }^{82}$ Während der vorrömischen Eisenzeit und der frühen Kaiserzeit, also in der Phase der römischen Einflussnahme und Besetzung wandelten sich die wandernden Dörfer in platzkonstante Siedlungen. Man interpretiert diese Anwesen als Sitze der Elite. Die Ähnlichkeit mit den jütländischen großen Rechteckanlagen ist tatsächlich erstaunlich. Beispiele sind die Gehöfte von Oss-Westerveld

82 Jansen / Fokkens 2010. 


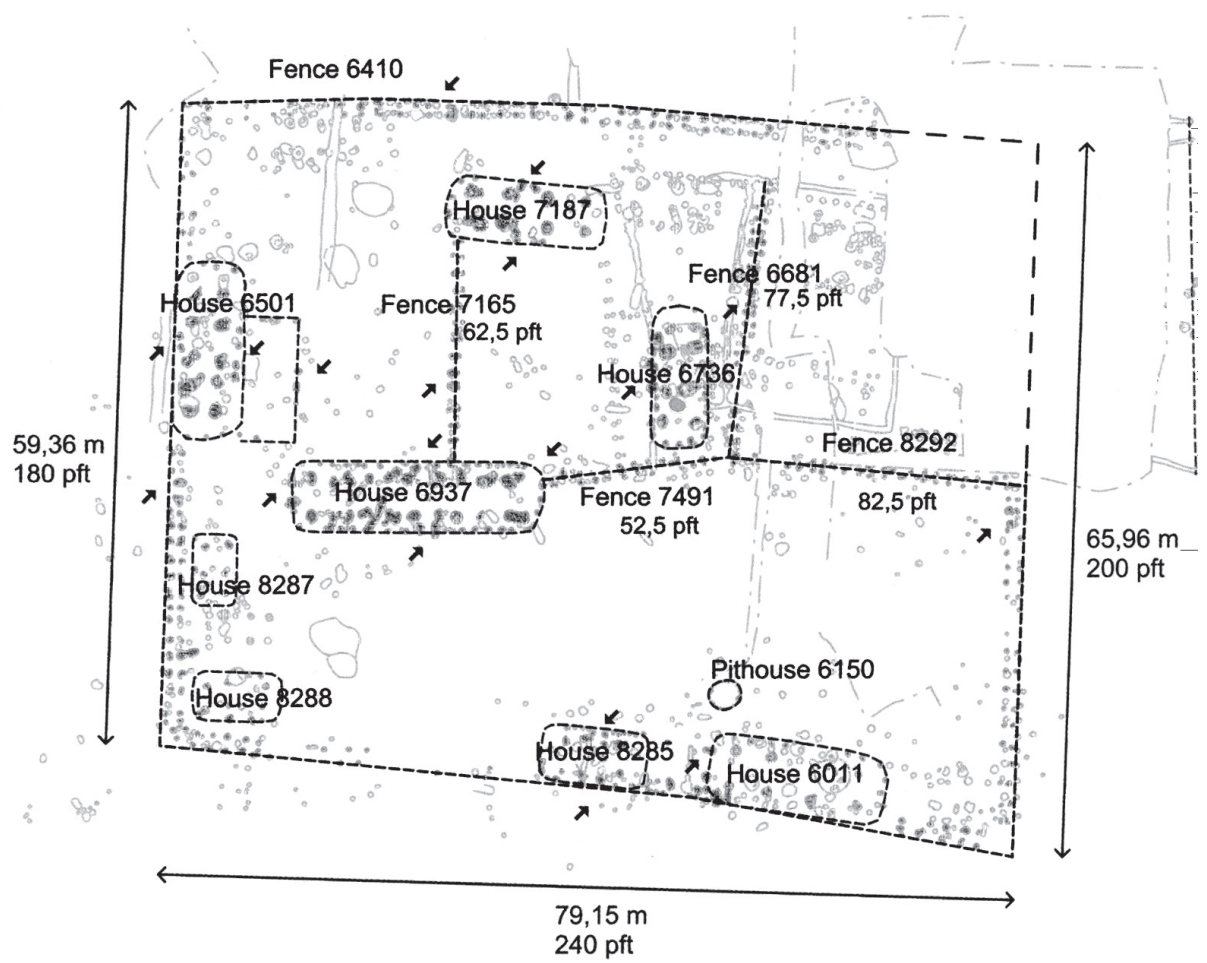

Abb. 11: Tjørring, Jütland. „Fürstliches“ Gehöft (nach Møller-Jensen 2010, S. 203 Fig. 6 - verändert).

(gegraben 1976-1986) ${ }^{83}$ mit den Abmessungen 260 auf 380 m bzw. 7,5 ha und zweifacher oder dreifacher rechteckiger Palisade, sowie auch Oss-Schalskamp ${ }^{84}$ mit den Abmessungen 150-180 m bzw. 2,6 ha und Palisadenumzäunung mit abgerundeten Ecken. Oss-Westerfeld weist fünf Bauphasen (von 25 v. Chr. bis 150 n. Chr.) mit wechselnder Lage der schließlich rund 30 Häuser auf. Ein Herrenhof mit einem römisch beeinflussten sog. Portikus-Haus hebt sich jeweils in der Südwestecke der Anlage heraus, in dem vor allem auch römische Keramik und Weinamphoren gefunden worden sind. Das Ganze wird als proto-villa bezeichnet, bewohnt von einem lokalen Anführer, dessen Ansehen auf alten Stammes- oder Familientraditionen beruhte und der den Kontakt zur römischen Herrschaft kontrollierte. Oss-Schalskamp, nur 400 m von Westerfeld entfernt, umschließt zweischiffige Wohn-Stall-Häuser aus der ersten Hälfte des 1. Jahrhunderts n. Chr., ist also zeitgleich mit Oss-Westerfeld. Man kann eine Hierarchie der Siedlungen registrieren. ${ }^{85}$

83 Jansen / Fokkens 2010, S. 135 f. mit Fig. 2 und 3.

84 Jansen / Fokkens 2010, S. 137 mit Fig. 4.

85 Jansen / Fokkens 2010, S. 140 Fig. 7. 


\section{Die Strukturierung des Naturraums}

\subsection{Hierarchien in der Landschaft}

Die Abbildung von Hierarchien der Siedlungen in der Landschaft versucht man auf verschiedenen Wegen zu erkennen. Ausgrabungsbefunde spiegeln die unterschiedliche Ranghöhe der Siedlungen. Der aber noch begrenzte Ausgrabungs- bzw. Publikationsstand zwingt auch bei dieser Frage dazu, Ergebnisse aus verschiedenen Landschaften zusammenzusehen und damit zu verallgemeinern.

Die Elemente im Siedlungsnetz sind kleine Gehöftansammlungen und größere Dörfer, die Strukturen unterschiedlichen Ranges innerhalb der Siedlungen, darunter sind sog. Herrenhöfe, und auch Befestigungsanlagen. Die Dichte der bäuerlich-landwirtschaftlich ausgerichteten Gehöftkomplexe ist - wie die Karten zu Anfang zeigen - beachtlich; tatsächlich liegen die Siedlungen - wie es die Regel ist über die Zeiten hinweg - im Abstand von wenigen Kilometern, die „Gemarkungen“ mit Radius von $2 \mathrm{~km}$ stoßen aneinander. Verblüffend fast flächendeckend ist auch die Verbreitungsdichte von bestimmten Keramikformen der frühen römischen Kaiserzeit, die auch ohne Dorfgrabungen Siedlungsplätze eindeutig zu umschreiben erlaubt. ${ }^{86}$

Die Abfolge der Vormarschlager in der Germania wie bei Hedemünden sind zugleich Hinweise auf die Bevölkerungsdichte in der Germania und damit auch auf die militärische Stärke der Stammesgruppen. Über die Bevölkerungsdichte habe ich bei der früheren Tagung 2004/2007 in Osnabrück berichtet und eigene statistische Überlegungen dazu angestellt. Inzwischen gibt es neue Fakten, die zu vergleichbaren Ergebnissen führen, was die Bevölkerungsdichte angeht - in diesem Fall in den römischen Provinzen am Rhein. ${ }^{87}$ Der Aufsatz betrachtet sich als Beitrag zur Landschaftsarchäologie, hat also flächendeckend die Siedlungsnetze im Blick.

Nico Roymans hat zudem für den kriegerischen Stamm der Bataver errechnet, dass die Gruppe aus nur etwa 40000 Menschen bestand, die immerhin aber 5000 Krieger für römische Auxiliareinheiten zu stellen hatten, was ein Verhältnis von 8:1 bedeutet. $^{88}$

Die erste Möglichkeit, Hierarchien zu erschließen, ist, dass man die bisher bekannten sog. Elite- oder Fürstengräber - die sichtlich eine Oberschicht und Herrschaft spiegeln - kartiert und mit Hilfe graphischer Methoden dann die Landschaft unterteilt (z.B. mit den sog. Thiessen-Polygonen oder der Methode der DelaunayTriangulation). Das kann aber nur ein momentanes Zufallsbild sein, was sich immer dann ändert, wenn neue ranghohe Fundkomplexe entdeckt werden. Ich habe das

86 Meyer 2009, S. 60 Abb. 3, S. 62 Abb. 6 Karten.

87 Wendt / Zimmermann 2009 mit Berichten zur Bevölkerungsdichte im Rheinland: 640000 bis 1,3 Millionen, auf $131850 \mathrm{~km}^{2}$ - Bevölkerungsdichte 4,8-10 Einwohner auf dem km²; vgl. höhere Zahlen: Steuer 2007, S. 358 bis zu 60 Einwohner pro km².

88 Roymans 2009, S. 86; in Bezug auf 50\% Männer 1:4. 
schlicht mit einfacher Kartierung für Norddeutschland versucht und bin zu dem Ergebnis gekommen, dass gegenwärtig das Herrschaftsgebiet einen Durchmesser von 20/30 bis höchstens $50 \mathrm{~km}$ hatte. Für Jütland ist das ebenfalls versucht worden, und man ist auf ähnliche Abstände bzw. Durchmesser von Herrschaftsgebieten gekommen. ${ }^{89}$ Michael Gebühr hat gezeigt, dass zur frühen Römischen Kaiserzeit das Verhältnis normale Personen zu einen „Fürst“ 1: 50 bis 1:300 betrug, in der späten Kaiserzeit dann aber 1:1000. ${ }^{90}$

Den Charakter und die Ranghöhe von Siedlungen oder Gehöften der frühen Römischen Kaiserzeit in der Germania möchte man gern durch den direkten Vergleich von Hausgrößen mit nahebei gelegenen „fürstlichen“ Bestattungen erschließen. Nur ist das bisher kaum gelungen. Genannt wurden schon die Befunde zu Tjørring mit rechteckigen Großgehöften und dabei liegenden reichen Gräbern. Gegenwärtig wird eine Siedlung bei Hitzacker im Kr. Lüchow-Dannenberg ausgegraben und ausgewertet, in dessen unmittelbarer Nähe - ein Zusammenhang besteht ohne Zweifel - die drei bekannten „Fürstengräber“ von Marwedel vor Jahren schon ausgegraben worden sind, die sich auf etwa zwei Generationen verteilen. ${ }^{91}$ Nachgewiesen sind jetzt Hausgrundrisse, die sich aber grundsätzlich kaum von anderen größeren Gehöften unterscheiden. Das Besondere ist hier übrigens wieder die Konzentration von Eisengewinnung auf demselben Areal. ${ }^{92}$

Ein zweiter Weg bietet sich über die Einbeziehung des Naturraumes an. Auch dies ist vor allem für Jütland versucht worden, weil dort tief ins Land reichende Flusstäler/Fjorde die Halbinsel gliedern. Außerdem gehört dann zu jeder Landschaftseinheit - doch erst in jüngerer Zeit - meist ein Platz für Kriegsbeuteopfer. Oder die Landschaftsteile sind zusätzlich mit Befestigungen, Palisadenzügen, Wällen und Gräben abgeriegelt. Bisher kam man mit den Datierungen nicht weiter als bis ins frühe 3 . Jahrhundert zurück, so beim Olgerdiget. ${ }^{93}$ Doch neue Ausgrabungen haben differenziertere Aussagen geliefert, für den Olgerdiget eine erste Phase um 50 n. Chr., eine zweite um 70 n. Chr. und eine dritte um 90 n. Chr. ${ }^{94}$

Dem entspricht die Erwähnung bei Tacitus, der eine solche Befestigung für die ältere Römische Kaiserzeit nennt, den Angrivarierwall an der Grenze des Gebietes der Cherusker. ${ }^{95}$

Der dritte Weg wäre, aber mit noch größerer statistischer Unsicherheit belegt, die Kartierung von Siedlungen mit Herrenhöfen, was aber wiederum höchstens in Anfangsversuchen für Jütland möglich wäre. Denn noch geht die Diskussion darum, ob von einem Herrenhof, einer magnate’s farmstead, nur innerhalb einer größeren

89 Møller-Jensen 2010, S. 219 Fig. 24 nach Ethelberg 2003, S. 276 Fig. 152.

90 Gebühr 2009, S. 350 Abb. 7.

91 Laux 2001.

92 Nüsse 2007; 2009; 2014a,b; Bayer 2013.

93 Christensen 2003, S. 91 mit Karte Abb. 15.

94 Christensen 2006a; Ethelberg 2009, S. 175.

95 Tacitus, Ann. II 19. 
Dorfgemeinschaft Herrschaft ausgeübt werden konnte, der ,Herrscher' dort vielleicht aber auch nur als primus inter pares zu sehen ist, oder ob man Plätze erkennen kann, in denen tatsächlich ein überregional wirkender Germane, Anführer, Adliger residiert hat. Per Ethelberg hat versucht, für das südliche Jütland solche Zentralorte der Herrschaft und damit die Größe der Territorien zu beschreiben (Abb. 12), ${ }^{96}$ mit dem Ziel, das Gebiet früher Herrschaftszentren zu definieren, die erkennbar seien aufgrund von Unterschieden im Hausbau und den Sachgütern. Ähnlich ist der Versuch, noch großräumiger Jütland insgesamt zu erfassen, dieser stützt sich auf die Verteilung der Waffenopferfunde und der ungewöhnlich reichen Gräber, mit dem Ziel, frühe „Königreiche“ als Machtkonzentrationen abzugrenzen. ${ }^{97}$

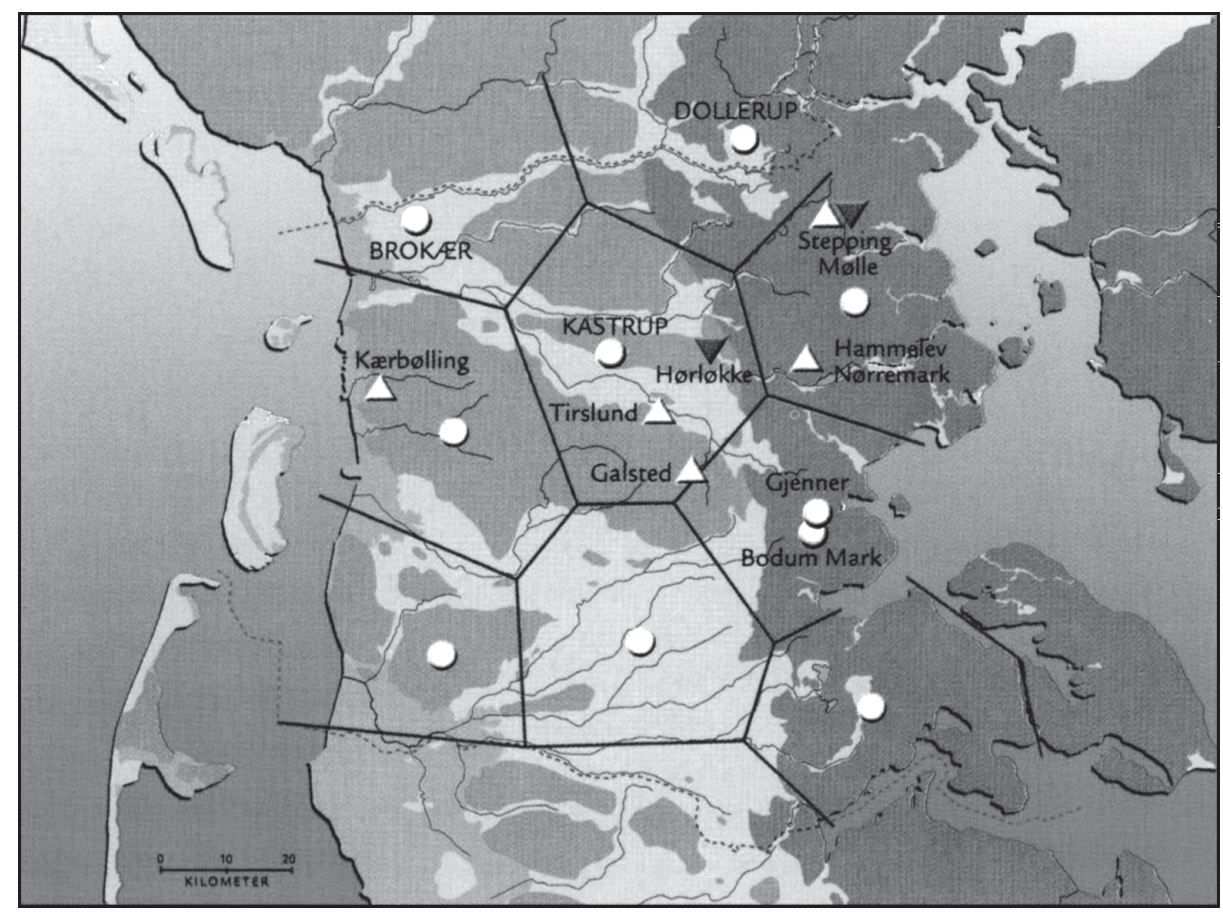

Abb. 12: „Fürstengräber“ und Herrenhöfe der Römischen Kaiserzeit im südlichen Jütland (nach Møller-Jensen 2010, S.219 Fig. 24 - übernommen von Ethelberg 2003, S. 276 Fig. 152).

96 Ethelberg 2003, S. 276 Fig. 152: „Fürstengräber“, Häuptlingsgehöfte und Häuptlingsgräber im südlichen Jütland; aufgeschlüsselt nach Thiessen-Polygonen.

97 Ethelberg 2009, S. 177 Abb. 9. 


\subsection{Landschaftsarchäologie}

Die archäologische Forschung hat Landschaftsgeschichte mit Umwelt und Besiedlung seit langem regelhaft im Blick, auch für die Römische Kaiserzeit in der Germania, teils von der Archäologie ${ }^{98}$ ausgehend, teils von der Biologie und Geologie. ${ }^{99}$

Eine relativ neue Forschungsrichtung wird unter dem Begriff „Landschaftsarchäologie“ zusammengefasst, ${ }^{100}$ wobei es nicht allein darum geht, alle Siedlungsrelikte, Gräberfelder und Wirtschaftsanlagen sowie Ackerfluren in einem Gebiet zu erfassen, sondern auch ihren Zusammenhang zu beschreiben, um abzulesen, wie bewusst die Strukturierung der Landschaft vorgenommen worden ist; manche sagen sogar, dass Landschaft inszeniert worden sei. Eine erstaunliche breite Methoden-Diskussion ist ausgebrochen - verknüpft mit der Benennung „spatial turn“, wobei die Begriffe Landschaft und Umwelt verbunden werden. ${ }^{101}$ Manchmal werden auch Beispiele genannt, aber gegenwärtig bleibt es noch zumeist beim Theoretischen. Breite Versuche hat es für prähistorische Epochen gegeben, für die Römische Kaiserzeit weniger. $^{102}$

Mit statistischen Verfahren wie die Errechnung der sog. Thiessen-Polygone oder der sog. Delaunay-Triangulation erfassen die über Geographische Informationssysteme (GIS) gewonnenen Daten eine Landschaft mehr oder weniger komplett, und ermöglichen Voraussagen zu noch nicht entdeckten Siedlungsplätzen; es lassen sich Prognosekarten erstellen. ${ }^{103}$

Über eine erkannte Hierarchie der Siedlungen z.B. mit Herrensitzen ergibt sich die Projektion der Herrschaft in die Landschaft hinein. Es geht um die Abhängigkeit von Höfen und Dörfern vom Herrenhof oder Adelsitz, ${ }^{104}$ es geht um die Kenntnis der Netzwerke. ${ }^{105}$

Das sind erste Ansätze einer politisch-sozialen Gliederung der Gesellschaft in der Germania. In der späten vorrömischen Eisenzeit und in der frühen römischen Kaiserzeit ist der Beginn dieser Vorgänge über archäologische Befunde zu erfassen. Das ist

\footnotetext{
98 Zum Beispiel Jankuhn 1977.
}

99 Behre 2008.

100 Steuer 2001, S. 630 f.; verschiedene Auffassungen gibt es aber, was unter „Landschaftsarchäologie“ zu verstehen ist, was hier nicht näher besprochen werden kann.

101 Genannt seien, jeweils mit weiterführender Literatur: Gramsch 2003; Brather 2006, 2011; Meier 2009; Knopf 2013; Müller-Scheessel 2013, S. 118 f. zum „spatial turn“.

102 Vgl. schon den Tagungsband „Landscape and Society“ von 1999; Brather 2006, S. 84 f. stellt z.B. die Graduiertenkollegs der DFG zu Siedlungs- und Landschaftsarchäologie in einer Tabelle zusammen, in der nur ein Programm auch die Zeit um Chr. Geb. berücksichtigt (Kelten, Germanen, Römer im Mittelgebirgsraum zwischen Luxemburg und Thüringen 1993-1999).

103 Steuer 2006, S. 163; Müller-Scheessel 2013, S. 111 f., S. 120 f.

104 Skre 1999, S. 420 Abb. 4: Von den einzelnen Großhöfen bis um 200 n. Chr. zur Hierarchie von Großhöfen mit abhängigen Höfen in den nachfolgenden Jahrhunderten: Entstehung eines Netzwerkes. $105 \mathrm{Zu}$ diesem Ansatz bei besserer Quellenlage: Müller 2009. 
ein Prozess, der damals begann und bis zur Karolinger- bzw. Wikingerzeit zu verfolgen ist.

Die dichte Lage der dörflichen Siedlungen - was die Anfangsthese stützt wird inzwischen anhand statistischer Verfahren mit voraussagbaren Ergebnissen bestätigt, ${ }^{106}$ über die man über die intensive Prospektion hinaus errechnen kann, wo ein weiterer Siedlungsplatz zu erwarten ist, den man dann leicht dokumentieren kann.

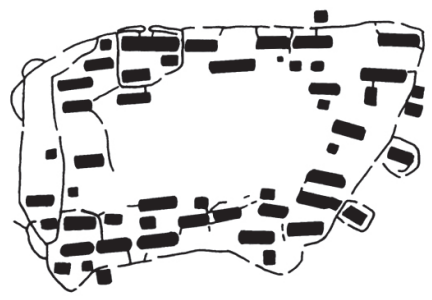

1st c. B. C. HODDE

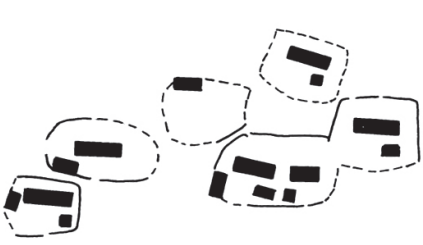

1st - 2nd c. A. D.
FREDERIKSDALVEJ,
RANDERS

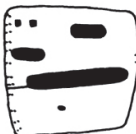

4th - 5th c. A.D.

VORBASSE

Abb. 13a: Größenentwicklung der Gehöfte als Abbild der wachsenden wirtschaftlichen Kraft von der vorrömischen Eisenzeit über die Römische Kaiserzeit bis zum frühen Mittelalter (nach Kaldal Mikkelsen 1999, S. 190 Fig. 10 - Ausschnitt), oder (Hvass 1993, S. 189 und S. 190 Abb.

- jeweils obere Hälfte).

Die während der späten vorrömischen Eisenzeit und frühen Römischen Kaiserzeit noch relativ kleinen landwirtschaftlich ausgerichteten Gehöfte wachsen bis ins Mittelalter ständig an, und zwar in den über viele Jahrhunderte existierenden und sich wandelnden Siedlungen, allein wenn man die Grundrisse der Wohn-Stall-Häuser und den Umfang der Stall-Teile für das Vieh betrachtet sowie die Parzellengröße der Gehöfte mit den Nebenbauten selbst. Einige graphische Zusammenstellungen haben das in den letzten Jahren mehrfach überzeugend gezeigt (Abb. 13a,b). ${ }^{107}$ Am Ende

106 Steuer 2006, S. 161-164.

107 Hvass 1993, S. 189 f. Abb.; Riedel 1999, S. 81 Fig.1; Kaldal Mikkelsen 1999, S. 187 ff. mit den Fig. 8, 10 und 11; Siedlungs-, Gehöft- und Hausformen 2005, S. 304 Abb. 59, S. 305 Abb. 60. 
steht das, was als Grundherrschaft mit Haupthof und abhängigen Höfen bezeichnet wird, wie im frühmittelalterlichen Tissø auf Seeland. ${ }^{108}$ Die Wurzeln in der Germania liegen in der römischen Kaiserzeit. Wie weit das auf innere gesellschaftliche Dynamik zurückging und bzw. oder auch auf Einflüsse aus der römischen Welt, muss noch diskutiert werden.

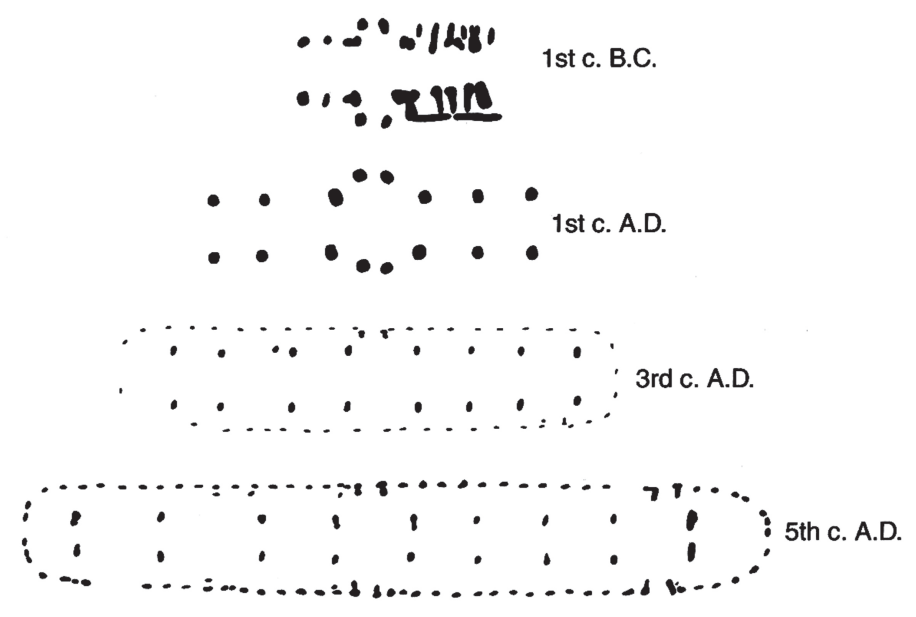

Abb. 13b: Größenentwicklung der Gehöfte als Abbild der wachsenden wirtschaftlichen Kraft von der vorrömischen Eisenzeit über die Römische Kaiserzeit bis zum frühen Mittelalter (nach Kaldal Mikkelsen 1999, S. 190 Fig. 10 - Ausschnitt), oder (Hvass 1993, S. 189 und S. 190 Abb. - jeweils obere Hälfte).

\subsection{Wege}

Ein weiteres Element der Strukturierung der Landschaft sind die Verkehrswege, zu Lande und auf den Gewässern, zwischen den einzelnen Siedlungen sowie über die Landschaften hinweg. ${ }^{109}$

Gegenüber den toposartigen Schilderungen von den undurchdringlichen Wald- und Sumpfgebieten in der Germania ist inzwischen ein anderes Bild zu entwerfen:

- Die Landschaften sind gerodet, offen und mit einem dichten Netz von Siedlungen überzogen, für die Wegeverbindungen vorauszusetzen sind.

- Sorgfältig gebaute Bohlenwege über die großen Moorflächen wurden schon seit der Bronzezeit und kontinuierlich immer wieder neu bis ins Mittelalter über die Römische Kaiserzeit hinweg eingerichtet oder alt ausgebaut. Und das ist nur

108 Jørgensen 2005.

109 Jöns 2009; Schlüter 2007. 
dann - wie früher schon formuliert ${ }^{110}$ - verständlich, wenn man davon ausgeht, dass auf dem trockenen Land ebenfalls gut befahrbare Wege unterhalten wurden. Ich gehe auch davon aus, dass die pontes longi, die Bohlenwege, die Germanicus erwähnt, baut und instand setzt, germanische Verkehrsanlagen waren, die natürlich vom römischen Militär ebenfalls genutzt worden sind. ${ }^{111}$

- Im Zuge der Erforschung römischer Marschlager in der Germania aus der Zeit der frühen Germanenkriege ist es heute möglich, Wegeverbindungen über die verlorengegangenen römischen Sandalennägel zu kartieren, wie Klaus Grote für das Umfeld von Hedemünden eindrucksvoll gezeigt hat. ${ }^{112}$ Es ergeben sich natürliche, sinnvolle Trassen, die man schon vorher kannte, die aber nun durch den Marsch römischen Militärs wieder bestätigt worden sind. Die Truppenteile marschierten eben dort entlang, wo solche Wegeführungen bestanden. Es ist bekannt, dass der Marschweg des Varus am Fuße des Kalkrieser Berges entlang ebenfalls eine wichtige Überlandverbindung war, die schon vorher und später bis ins Mittelalter genutzt wurde. Es ist bei derartigen Kriegen denn auch üblich, beim Marsch auf den Wegen durch die Dörfer sich aus dem Raum heraus zu versorgen und diese Dörfer dann zu vernichten. Immer wieder flüchteten sich die Bewohner mit ihrem Vieh in die Wälder, wohin keine offenen Wege führten.

\section{Nachbemerkungen}

Mehrfach habe ich gesagt, dass die germanischen Landschaften zwischen Rhein und Elbe und weiter nach Osten und Norden hinaus dicht besiedelt waren, der Wald durchaus gerodet, und Mitteleuropa eben nicht eine fremde, ferne Welt war. Vielmehr gibt es wohl starke Ähnlichkeiten zur frühgeschichtlichen Besiedlung wie in der Merowinger- und Karolingerzeit. Sicherlich hat die archäologische Forschung von diesen Siedlungen erst wenige Prozent gefunden und erforscht, aber immerhin geht die Zahl der dokumentierten Hausgrundrisse von den Niederlanden über Norddeutschland bis nach Jütland in die Tausende, ${ }^{113}$ und inzwischen sind schon einige hundert Ansiedlungen großflächig bis komplett ausgegraben. ${ }^{114}$ Es gibt dazu erste umfangreiche Zusammenstellungen. So wird es möglich, die Organisation und Strukturierung der weiten Landschaft, die Siedlungsnetze und die Planung der Dörfer flächendeckend

110 Steuer 2007, S. 338.

111 Tacitus, Ann. I, 61 und 63.

112 Grote 2012, S. 310 Abb. 325.

113 Brabandt 1993, S. 11: 900 Grundrisse von Langhäusern aus der Germania magna; Nüsse 2014: rund 2000 Grundrisse kaiserzeitlicher und frühvölkerwanderungszeitlicher Wohngebäude; gegenwärtig sind aus einzelnen Siedlungen jeweils allein schon um die 100 Häuser erfaßt: Bock 2013, S. 211. 114 Nüsse 2010. 
zu beschreiben - zumindest modellhaft. Doch können in diesem kurzen Beitrag nur ausgewählte Beispiele genannt und beschrieben werden.

Regionalanalysen werden seit langem versucht, so für Schleswig-Holstein mit Angeln von Herbert Jankuhn schon 1977. ${ }^{115}$ Seine Kartenbilder sind heute nach der Vermehrung der Fundstellen so nicht mehr zu verwenden, doch ist der methodische Ansatz weiterführend gewesen. Die Siedlungskammer Flögeln-Eekhöljen im Ldkr. Cuxhaven ${ }^{116}$ mit einer Größe von 23 km² $^{2}$ ist über Jahre fast vollständig erforscht worden, einschließlich der kaiserzeitlichen Ackerfluren, ebenso die benachbarte Siedlungskammer um Loxstedt ${ }^{117}$ oder neu auch der Raum um Sievern als Zentralplatz. ${ }^{118}$ Auf der Insel Sylt galten schon früh Forschungen nicht nur der größeren ausgegrabenen Siedlung Archsum, sondern auch dem Umfeld. ${ }^{119}$ Und nicht zuletzt ist von Klaus Grote - wie anfangs gezeigt - der Siedlungsraum um das Lager Hedemünden, eingebunden in den Naturraum, systematisch erschlossen worden. ${ }^{120}$

\section{Literatur}

RGA Reallexikon der Germanischen Altertumskunde Bd. 1 (Berlin / New York 1973) - Bd. 35 (Berlin / New York 2007).

Aufderhaar, Iris / Bittmann, Felix / Brandt, Imke / Jöns Hauke / Klein, Christina /Schön, Matthias D. / Stümpel, Harald / Wolters, Steffen / Zimmermann, W. Haio: Neue Forschungen am Zentralplatz von Sievern, Lkr. Cuxhaven. Germania 87, 2009 (2011), S. 173-220.

Bayer, Yvonne: „Das Haus auf dem Hügel... “ - Die Ausgrabungen von Hitzacker-Marwedel 2010 und 2011, in: Immo Heske / Hans-Jürgen Nüsse / Jens Schneeweiß (Hrsg.), Landschaft, Besiedlung und Siedlung. Archäologische Studien im nordeuropäischen Kontext. Festschrift für Karl-Heinz Willroth zu seinem 65. Geburtstag. Göttinger Schriften zur Vor- und Frühgeschichte Bd. 33 (Neumünster / Hamburg 2013) S. 191-200.

Beck, Heinrich / Steuer, Heiko (Hrsg.): Haus und Hof in ur- und frühgeschichtlicher Zeit. Bericht über zwei Kolloquien der Kommission für die Altertumskunde Mittel- und Nordeuropas vom 24. bis 26. Mai 1990 und 20. bis 22. November 1991 (34. und 35. Arbeitstagung) (Gedenkschrift für Herbert Jankuhn). Abh. Akad. Wiss. in Göttingen, Phil.-Hist. Kl. Dritte Folge Nr. 218 (Göttingen 1997).

Bederkesa 2010: Gedächtnis-Kolloquium Werner Haarnagel (1907-1984). Herrenhöfe und die Hierarchie der Macht im Raum südlich und östlich der Nordsee von der Vorrömischen Eisenzeit bis zum frühen Mittelalter und zur Wikingerzeit. 11.-13. Oktober 2001 Burg Bederkesa in Bad Bederkesa. Siedlungs- und Küstenforschung im südlichen Nordseegebiet Bd. 33 (Rahden/ Westf. 2010).

115 Jankuhn 1977.

116 Zimmermann 1995, S. 207 mit Abb. 32.

117 Zimmermann 1997, S. 448 Abb. 9.

118 Aufderhaar et al. 2009 (2011) S. 174 ff. mit Abb. 1 (Hauke Jöns).

119 Kossack 1997, dazu Steuer 2007, S. 353 und S. 355 mit Abb. 9; auch Lütjens 2006 zur Phase der jüngeren vorrömischen Eisenzeit und der frühen römischen Kaiserzeit (Ergebnisse der Dissertation 2005); Lütjens 2008.

120 Grote 2012. 
Behre, Karl-Ernst: Landschaftsgeschichte Norddeutschlands. Umwelt und Siedlung von der Steinzeit bis zur Gegenwart (Neumünster 2008).

Berg-Hobohm, Stefanie: Herrenhöfe in Bayern - Die rechteckigen Grabenwerke der frühen Eisenzeit, in: Bederkesa 2010, S. 95-106.

Bock, Jan: Der kaiserzeitliche und völkerwanderungszeitliche Siedlungsplatz Groß Meckelsen, Ldkr. Rotenburg (Wümme) - Bedeutung und Perspektiven seiner Erforschung, in: Immo Heske / Hans-Jörg Nüsse / Jens Schneeweiß (Hrsg.), Landschaft, Besiedlung und Wirtschaft. Archäologische Studien im nordeuropäischen Kontext. Festschrift für Karl-Heinz Willroth zu seinem 65. Geburtstag. Göttinger Schriften zur Vor- und Frühgeschichte Bd. 33 (Neumüster 2013) S. 209-213.

Brabandt, J.: Hausbefunde der römischen Kaiserzeit im freien Germanien. Ein Forschungsstand. Veröffentlichungen des Landesamtes für archäologische Denkmalpflege Sachsen-Anhalt, Landesmuseum für Vorgeschichte Bd. 46 (Halle /S. 1993).

Brather, Sebastian: Entwicklungen der Siedlungsarchäologie. Auf dem Weg zu einer umfassenden Umwelt- und Landschaftsarchäologie? Siedlungsforschung. Archäologie - Geschichte Geographie 24, 2006, S. 51-97.

Brather, Sebastian: Siedlungs-, Umwelt- und Landschaftsarchäologie. Entdeckung und Analyse des Raumes. Freiburger Universitätsblätter 192, 2011, S. 123-136.

Brieske, Vera: Tinnumburg, RGA 30, 2005, S. 613-615.

Büchsenschütz, Olivier / von Nicolai, Renate: Gut geschützt im Viereck. Leben auf dem Lande in spätkeltischer Zeit, in: Die Welt der Kelten. Zentren der Macht - Kostbarkeiten der Kunst (Ostfildern 2012) S. 382-387.

Burmeister, Stefan / Wendowski-Schünemann, Andreas: Werner Haarnagel und der „Herrenhof“ der Feddersen Wierde - Anmerkungen zu einem sozialtopographischen Konzept, in: Bederkesa 2010, S. 35-52.

Caselitz, Peter / Schlüter, Wolfgang: Schädeltrophäen und Ahnenkult? Die menschlichen Skelettreste von der Pipinsburg bei Osterode/Harz. Studia Osteoarchaeologica 2 (Göttingen 2013).

Christensen, Lisbeth: Olgerdiget. RGA 22, 2003, S. 91-92.

Christensen, Lisbeth: Vestergård. RGA 32, 2006a, S. 295-296.

Chistensen, Lisbeth: Nye undersøgelser af Olgerdiget - en langvold fra jernalderern. Sonderjsyk Månedsskrift 2006(1)b, S. 3-10.

Dübner, Daniel: Neues zu Entwicklung und Gehöftstrukturen der kaiser- bis völkerwanderungszeitlichen Siedlung von Flögeln, Ldkr. Cuxhaven. Siedlungs- und Küstenforschung im südlichen Nordseegebiet Bd. 36 (Rahden/Westf. 2013), S. 225-233.

Eck, Werner: Die römischen Provinzen. Herrschaft und Verwaltungspraxis in der frühen Kaiserzeit, in: 2000 Jahre Varussschlacht - Imperium (Stuttgart 2009) S. 142-148.

Egeberg Hansen, Torben / Holst, Mads Kähler: Nørre Snede. RGA 21, 2002, S. 252-255.

Eisenschmidt, Silke: Die Ergebnisse einer größeren Siedlungsgrabung in Askov bei Vejen. Arkæologi i Slesvig / Archäologie in Schleswig 11, 2006, S. 59-66.

Eriksen, Palle / Rindel, Per Ole: Den vestjyske borg. Skalk 2005, Heft 1, S. 5-10.

Ethelberg, Per: The Chieftain's Farm of the Over Jerstal Group. Journal of Danish Archaeology 11, 1995, S. 111-136.

Ethelberg, Per: Haus und Siedlung der älteren römischen Kaiserzeit im ehemaligen Herzogtum Schleswig. Probleme der Küstenforschung im südlichen Nordseegebiet 27, 2001, S. 57-73.

Ethelberg, Per: Gården og landsbyen i jernalder og vikingetid (500 f.kr.-1000 e.kr.), in: Per Ethelberg / Nils Hardt / Bjørn Poulsen, Anne Brigitte Sørensen, Det Sønderjyske Landbrugs Historie. Jernalder, vikingetid og middelalder. Historisk samfund for Sønderjylland, Skrifter 82 (Haderslev 2003) S. 123-373. 
Ethelberg, Per: Verteidigungsanlagen und Haustypen der älteren römischen Kaiserzeit im ehemaligen Herzogtum Schleswig-Holstein, in: Christoph Grünewald / Torsten Capelle (Hrsg.), Innere Strukturen von Siedlungen und Gräberfeldern als Spiegel gesellschaftlicher Wirklichkeit? Akten des 57. Internationalen Sachsensymposiums 2006 (Münster 2007) S. 145-155.

Ethelberg, Per: Frühe Königreiche. Machtkonzentrationen in Südskandinavien im 1. bis 4. Jahrhundert n. Chr., in: 2000 Jahre Varusschlacht - Konflikt (Stuttgart 2009) S. 170-182.

Ethelberg, Per / Kruse, Pernille: Das Osterrönfeld-Haus: Status nach 10-jähriger Untersuchung. Arkæologi i Slesvig / Archäologie in Schleswig 14, 2013, S. 103-130.

Fried, Johannes: Karl der Grosse. Gewalt und Glaube. Eine Biographie (München 22014).

Gebühr, Michael: Reiche Bauern oder Fürsten? Germanische Eliten in der älteren römischen Kaiserzeit, in: 2000 Jahre Varusschlacht - Konflikt (Stuttgart 2009) S. 342-351.

Gramsch, A.: Landschaftsarchäologie: Ein fachgeschichtlicher Überblick und ein theoretisches Konzept, in: Jürgen Kunow / Johannes Müller (Hrsg.), Archäoprognose Brandenburg I: Symposium Landschaftsarchäologie und geographische Informationssysteme. Prognosekarten, Besiedlungsdynamik und prähistorische Raumordnung. Forschungen zur Archäologie Land Brandenburg 8 (Wünsdorf 2003) S. 35-54.

Grote, Klaus u.a.: Römerlager Hedemünden.Veröffentlichungen der archäologischen Sammlungen des Landesmuseums Hannover Bd. 53 (Dresden 2012).

Hårdh, Birgitta: Uppåkra. RGA 31, 2006, S. 516-520.

Harck, Ole: Archsum auf Sylt. Teil 3. Die Ausgrabungen in den römerzeitlichen Erdwerken Archsumburg, Tinnumburg und Trælbanken an der Westküste Schleswigs. RömischGermanische Forschungen Bd. 50 (Mainz 1990).

Harnecker, Joachim: Kalkriese war kein germanischer Urwald, in: Varusschlacht im Osnabrücker Land - Museum und Park Kalkriese (Mainz 2009) S. 89-91.

Heller, Erich: P. Cornelius Tacitus. Annalen. Lateinisch deutsch, hrsg. von Erich Heller. Reihe Tusculum (München und Zürich 1982).

Herschend, Frands: The origin of the hall in Southern Scandinavia. Tor 25, 1993, S. 175-199.

Herschend, Frands: Halle. RGA 13, 1999a, S. 414-425.

Herschend, Frands: Ordering landscapes, in: Settlement and Landscape 1999, S. 331.-335.

Holst, Mads Kähler: Inconstancy and stability - Large and small farmsteads in the village of Nørre Snede (Central Jutland) in the first millennium AD. Wandel und Beständigkeit - Große und kleine Höfe in der Siedlung Nørre Snede (Mitteljütland) im 1. Jahrtausend n. Chr., in: Bederkesa 2010, S. 155-179.

Hvass, Sten: Hodde - et vestjysk landsbysamfund fra ældre jernalder. Arkæologiske Studier 7 (København 1985).

Hvass, Sten: The Status of the Iron Age Settlement in Denmark, in: Beck / Steuer 1997, S. 377-413. Hvass, Sten: Settlement, in: St. Hvass / Birger Storgaard (Ed.), Digging into the Past. 25 Years of Archaeology in Denmark (Aarhus 1993) S. 187-194.

Hvass, Sten: Vorbasse. RGA 32, 2006, S. 595-599.

Jankuhn, Herbert: Einführung in die Siedlungsarchäologie (Berlin / New York 1977).

Jansen, Richard / Fokkens, Harry: Central places of the 1st and 2nd century AD in Maaskant region (Southern Netherlands), in: Bederkesa 2010, S. 133-146.

Jöns, Hauke: Iron production in Northern Germany during the Iron Age, in: Settlement and Landscape 1999, S. 249-260.

Jöns, Hauke: Zur ältesten Eisenverhüttung in Norddeutschland und im südlichen Skandinavien, in: Keltische Einflüsse 2007, S. 53-71.

Jöns, Hauke: Überlegungen zu Transport- und Kommunikationswegen des 1. Jahrtausends im nordwestdeutschen Nordseeküstengebiet, in: Sebastian Brather / Dieter Geuenich / Christoph 
Huth (Hrsg.), Historia archaeologica. Festschrift für Heiko Steuer zum 70. Geburtstag.

Ergänzungsbände zum RGA Bd. 70 (Berlin / New York 2009) S. 389-413.

Jöns, Hauke: Eisen und Macht - Gesellschaftliche Strukturen der Eisenökonomie von der Eisenzeit

bis zur Völkerwanderungszeit im Raum zwischen Mittelgebirge und Ostsee, in: Bederkesa 2010,

S. 107-118.

Jørgensen, Lars: Tissø. RGA 30, 2005, S.619-624.

Kaldal Mikkelsen, Dorthe: Single farm or village? Reflections on the settlement structure of the Iron

Age and the Viking period, in: Settlement and Landscape 1999, S. 177-193.

Kauffmann, Iris: Gristede. RGA 13, 1999, S. 59-60.

Kaul, Flemming: Vestervig - an Iron Age village mound in Thy, NW Jutland, in: Settlement and Landscape 1999, S. 53-66.

Kaul, Flemming: Priorsløkke. RGA 23, 2003, S. 462-466.

Kaul, Flemming: Vestervig. RGA 32, 2006, S. 296-302.

Keltische Einflüsse 2007: Sebastian Möllers / Wolfgang Schlüter / Susanne Sievers (Hrsg.), Keltische Einflüsse im nördlichen Mitteleuropa während der mittleren und jüngeren vorrömischen

Eisenzeit. Akten des Internationalen Kolloquiums in Osnabrück vom 29. März bis 1. April 2006.

Kolloquien zur Vor- und Frühgeschichte (Bonn 2007).

Knopf, Thomas: „Umwelt“ als Forschungsgegenstand: Konzepte und Theorien, in: Manfred K. H.

Eggert / Ulrich Veit (Hrsg.), Theorie in der Archäologie: Zur jüngeren Diskussion in Deutschland.

Tübinger Archäologische Taschenbücher Bd. 10 (Münster etc. 2013) S. 63-99.

Kossack, Georg: Dörfer im Nördlichen Germanien vornehmlich aus der römischen Kaiserzeit. Lage, Ortsplan, Betriebsgefüge und Gemeinschaftsform. Bayerische Akad. Wiss., Phil.-Hist. Kl. Abh. Neue Folge Heft 112 (München 1997).

Larsson, Lars (Ed.): Continuity for Centuries. A ceremonial building and its context at Uppåkra, southern Sweden. Uppåkrastudier 10. Acta Archaeologica Lundensia, Series in $8^{\circ}$, No. 48 (Stockholm 2004).

Larsson, Lars: Der Tempel von Uppåkra, in: CREDO. Christianisierung Europas im Mittelalter. Ausstellungskatalog Bd. 2 (Paderborn 2013) S. 325-326.

Laux, Friedrich: Marwedel. RGA Bd. 19, 2001, S. 371-374.

Løken, Trond: Oppkomsten av den germanske hallen. Hall og sal i eldre jernalder i Rogaland. Viking 64, 2001, S. 49-86.

Lütjens, Ingo: Archsum-Melenknop. Eine Siedlung der jüngeren Vorrömischen Eisen- und der frühen Römischen Kaiserzeit auf Sylt, Kr. Nordfriesland. Arkæologi i Slesvig / Archäologie in Schleswig 11, 2006, S. 39-44.

Lütjens, Ingo: Archsum LA 65 (Melenknop), Schichtpaket A. Universitätsforschungen zur prähistorischen Archäologie 149, 1 und 2 (Bonn 2008).

Lütjens, Ingo: „Klosterbakken B“ - Ein Reihendorf aus der frühen römischen Kaiserzeit. Arkæologi i Slesvig / Archäologie in Schleswig 12, 2008b, S. 105-120.

Magnus, Bente: Lofoten §. 2 Archäologisches. RGA 18, 2001, S. 567-569.

Martens, Jes: Fortified places in low-land Northern Europe and Scandínavia during the Pre-Roman-Iron Age, in: Keltische Einflüsse 2007, S. 87-105.

Martens, Jes: Vor den Römern. Eliten in der Vorrömischen Eisenzeit, in: 2000 Jahre Varussschlacht Konflikt (Stuttgart 2009) S. 334-341.

Martens, Jes: A magnate's farm at Borremose? Ein Herrenhof in Borremose?, in: Bederkesa 2010, S. 181-195.

Max, Jens: Im Fokus der Denkmalpflege, in: Archäologie in Deutschland 2002, Heft 1, S. 33-34.

Meier, Thomas: Umweltarchäologie - Landschaftsarchäologie, in: Historia archaeologica. Festschrift für Heiko Steuer zum 70. Geburtstag. Ergänzungsbände zum RGA Bd. 70 (Berlin / New York 2009) S. 697-734. 
Meyer, Michael: Der deutsche Mittelgebirgsraum um die Zeitenwende, in: 2000 Jahre Varusschlacht - Mythos (Stuttgart 2009) S.58-66.

Meyer, Michael (Hrsg.), Haus - Gehöft - Weiler - Dorf. Siedlungen der vorrömischen Eisenzeit im nördlichen Mitteleuropa. Berliner Archäologische Forschungen Bd. 9 (Rahden/Westf. 2010).

Mildenberger, Gerhard: Germanische Burgen. Veröff. der Altertumskommission Bd. VI (Münster Westfalen 1978).

Møller-Jensen, Erik: The „princely“ estate at Tjørring on Juland, in: Bederkesa 2010, S. 197-223.

Möllers, Sebastian: Die Schnippenburg bei Ostercappeln, Lkr. Osnabrück. Ein Zentralort mit Kultplatzfunktion im nordwestlichen Ausläufer der Mittelgebirgszone?, in: Keltische Einflüsse 2007, S. 1-45.

Müller-Scheessel, Nils: Mensch und Raum: Heutige Theorien und ihre Anwendung, in: Manfred K. H. Eggert, Ulrich Veit (Hrsg.), Theorie in der Archäologie: Zur jüngeren Diskussion in Deutschland. Tübinger Taschenbücher 10 (Münster u.a. 2013) S. 101-137.

Müller, Rosemarie: Die östliche Kontaktzone zwischen dem keltischen Kulturraum und dem Norden, in: Keltische Einflüsse 2007, S. 265-262.

Müller, Ulrich: Netzwerkanalysen in der Historischen Archäologie - Begriffe und Beispiele, in: Sebastian Brather / Dieter Geuenich / Christoph Huth (Hrsg.), Historia archaeologica. Festschrift für Heiko Steuer zum 70. Geburtstag. Ergänzungsbände zum RGA Bd. 70 (Berlin / Bew York 2009) S. 735-754.

Munch, Gerd Stamsø: Borg in Lofoten: a chieftains's farm in North Norway (Trondheim 2003).

Nørbach, Lars Christian: Organising iron production and settlement in Northwestern Europe during the Iron Age, in: Settlement and Landscape 1999, S. 237-247.

Nørbach, Lars Christian: Snorup § 1. Archäologisch. RGA 29, 2005, S. 178-181.

Nüsse, Hans-Jörg: Geomagnetische Prospektion und archäologische Untersuchungen bei den "Fürstengräbern" von Marwedel, Ldkr. Lüchow-Dannenberg. Praehistorische Zeitschrift 82, 2007, S. 85-113.

Nüsse, Hans-Jörg: Alltägliches und Nichtalltägliches bei den „Fürsten von Marwedel“. Archäologie in Niedersachsen 12, 2009, S. 52-54.

Nüsse, Hans-Jörg: Haus, Gehöft und Siedlung im Norden und Westen der Germania magna.

Nüsse, Hans-Jörg: Die „Fürsten von Marwedel. Söldner im Dienste des römischen Kaisers. Archäologie in Niedersachsen 17, 2014, S. 68-71.

Pape, Jürgen: Die germanische Siedlung von Engter, in: Wolfgang Schlüter (Hrsg.), Kalkriese Römer im Osnabrücker Land. Archäologische Forschungen zur Varusschlacht (Bramsche 1993) S. 307-321.

Rindel, Per Ole: Development of the village community 500 BC - 100 AD in west Jutland, Denmark, in: Settlement and Landscape 1999, S. 79-99.

Rindel, Per Ole: Lyngsmose. RGA 19, 2001, S. 69-71.

Roymans, Nico: Die Bataver. Entstehung eines Soldatenvolkes, in: 2000 Jahre Varusschlacht Mythos, hrsg. vom Landschaftsverband Lippe (Stuttgart 2009) S. 85-98.

Schäfer, Andreas / Schroth, Bernhard: Das Fragment einer vergoldeten Großplastik aus WetzlarDalheim, Lahn-Dill-Kreis. Hessen Archäologie 2008 (2009), S. 71-73.

Schlüter, Wolfgang: Verkehrswege und Verkehrsräume der jüngeren Bronze- und der vorrömischen Eisenzeit in Nordwesdeutschland und den nördlichen Niederlanden, in: Keltische Einflüsse 2007, S. 293-310.

Schlüter, Wolfgang / Wiegels, Rainer: Kalkriese. RGA 16, 2000, S. 180-199.

Schlüter, Wolfgang: Pipinsburg. RGA 23, 2003, S. 182-191.

Schlüter, Wolfgang: Schnippenburg. RGA 27, 2004, S. 228-233.

Schmid, Peter: Feddersen Wierde. RGA 8, 1994, S. 249-266.

Schmid, Peter: Heidenschanze. RGA 14, 1999a, S. 154-156. 
Schmid, Peter: Heidenstadt. RGA 14, 1999b, S. 156.

Schmid, Peter: Der „Herrenhof“ der Feddersen Wierde, in: Bederkesa 2010, S. 21-34.

Schnurbein, Siegmar von: Aus dem augusteischen Stützpunkt Waldgirmes verschleppte Funde, in: Angelika Abegg / Dörte Walter / Susanne Biegert (Hrsg.), Die Germanen und der Limes. Ausgrabungen im Vorfeld des Wetterau- Limes im Raum Wetzlar-Gießen. Römisch-Germanische Forschungen 67 (Mainz 2011) S. 95-97.

Schön, Matthias D.: Die Heidenschanze bei Sievern. Eine fast 2000 Jahre alte Befestigung. Archäologie in Niedersachsen 3, 2000, S. 57-59.

Schuster, Jan: Herzsprung. Eine kaiserzeitliche bis völkerwanderungszeitliche Siedlung in der Uckermark. Berliner Archäologische Forschungen Bd. 1 (Rahden/Westf. 2004).

Settlement and Landscape 1999: Charlotte Fabech / Jytte Ringtved (Ed.), Settlement and Landscape. Proceedings of a conference in Århus, Denmark, May 4-7 1998 (Aarhus 1999).

Sicherl, Bernhard: Eisenzeitliche Befestigungen in Westfalen, in: Keltische Einflüsse 2007, S. 107-151.

Siedlungs-, Gehöft- und Hausformen. RGA 28, 2005, S. 282-319., darin: I. Norwegen S. 282-293 (Trond Løken), II. Schweden S. 293-301 (Lennart Carlie), III. Dänemark, S. $301-314$ (Jørgen Lund), IV. Kontinent, S. 314-319 (Heiko Steuer).

Skree, Dagfinn: Aristocratic dominion and landownership in Norway 200-1100 AD, in: Settlement and Landscape 1999, S. 415-422.

Steinmetz, Wolf-Dieter: Dynamisches Geschehen - viel Geschichte. Die Ausgrabungen 2006-2012 auf dem Burgwall „Schwedenschanze“ bei Isingerode. Archäologie in Niedersachsen 16, 2013. S. 54-57.

Steuer, Heiko: Landschaftsarchäologie. RGA 17, 2001, S. 630-634.

Steuer, Heiko: Verbreitungskarte. RGA 32, 2006, S. 142-166.

Steuer, Heiko: Besiedlungsdichte, Bevölkerungsgrößen und Heeresstärken während der älteren Römischen Kaiserzeit in der Germania magna, in: Gustav Adolf Lehmann / Rainer Wiegels (Hrsg.), Römische Präsenz und Herrschaft im Germanien der augusteischen Zeit. Der Fundplatz von Kalkriese im Kontext neuer Forschungen und Ausgrabungsbefunde. Beiträge zur Tagung in Osnabrück vom 10. bis 12. Juni 2004. Abh. Akad. Wiss. zu Göttingen, Phil.-Hist. Kl. Dritte Folge Bd. 279 (Göttingen 2007) S. 337-362.

Tempel, Wolf-Dieter: Die Ausgrabung einer bäuerlichen Siedlung bei Groß Meckelsen, Kr. Rotenburg/W., in: Werner Budesheim / Horst Keiling (Hrsg.), Zur Geschichte und Archäologie der Germanen zwischen Rhein und Oder um die Zeitenwende. Freie Lauenburgische Akademie für Wissenschaft und Kultur / Beiträge für Wissenschaft und Kultur Bd. 6 (Hamburg 2003) S. 141-156.

Tempel, Wolf-Dieter: Eine Dorfsiedlung der römischen Kaiserzeit und Völkerwanderungszeit bei Groß Meckelsen Ldkr. Rotenburg (Wümme), in: Mahmoud Fanza / Henning Haßmann (Hrsg.), Archäologie - Land - Niedersachsen. 25 Jahre Denkmalschutzgesetz - 400000 Jahre Geschichte (Stuttgart 2004) S. 429-435.

Verse, Frank: Die Befestigung auf dem „Oberwald“ bei Greifenstein-Holzhausen, Lahn-Dill-Kreis. Profane Siedlung oder Kultplatz im peripheren Mittelgebirgsraum, in: Keltische Einflüsse 2007, S. 153-166.

Voss, Olfert, Drengsted. RGA 6, 1986, S. 177-180.

Waterbolk, Hjarm Talling, Zeijen. RGA 34, 2007, S. 486-497.

Wendt, Karl Peter / Zimmermann, Andreas: Bevölkerungsdichte und Landnutzung in den germanischen Provinzen des Römischen Reiches im 2. Jahrhundert n. Chr. Ein Beitrag zur Landschaftsarchäologie. Germania 86, 2008 (2009), S. 191-226.

Wieland, Günther: Viereckschanzen. RGA 32, 2006, S. 357-362, 
Zimmermann, W. Haio: Die Siedlungen des 1. bis 6. Jahrhunderts nach Christus von Flögeln-

Eekhöltjen, Niedersachsen. Die Bauformen und ihre Funktionen. Probleme der Küstenforschung im südlichen Nordseegebiet 19 (Hildsheim 1992).

Zimmermann, W. Haio: Flögeln. RGA 9, 1995a, S. 206-216.

Zimmermann, W. Haio: Haus, Hof und Siedlungsstruktur auf der Geest vom Neolithikum bis in das Mittelalter, in: Hans-Eckhard Dannenberg / Hein-Joachim Schulze (Hrsg.), Geschichte des Landes zwischen Elbe und Weser Bd.1 Vor- und Frühgeschichte (Stade 1995b) S. 251-288.

Zimmermann, W. Haio: Haus, Hof und Siedlungsstruktur auf der Geest vom Neolithikum bis in das Mittelalter im Elbe-Weser-Dreieck, in: Beck /Steuer 1997, S. 414 -460.

Zimmermann, W. Haio: Loxstedt. RGA 18, 2001, S. 629-633. 
